Internet Engineering Task Force (IETF)

M. Jones

Request for Comments: 7517

Category: Standards Track

Microsoft

ISSN : 2070-1721

May 2015

JSON Web Key (JWK)

Abstract

A JSON Web Key (JWK) is a JavaScript Object Notation (JSON) data structure that represents a cryptographic key. This specification also defines a JWK Set JSON data structure that represents a set of JWKs. Cryptographic algorithms and identifiers for use with this specification are described in the separate JSON Web Algorithms (JWA) specification and IANA registries established by that specification.

Status of This Memo

This is an Internet Standards Track document.

This document is a product of the Internet Engineering Task Force (IETF). It represents the consensus of the IETF community. It has received public review and has been approved for publication by the Internet Engineering Steering Group (IESG). Further information on Internet Standards is available in section 2 of RFC 5741.

Information about the current status of this document, any errata, and how to provide feedback on it may be obtained at

http://www.rfc-editor.org/info/rfc7517.

Copyright Notice

Copyright (c) 2015 IETF Trust and the persons identified as the document authors. All rights reserved.

This document is subject to BCP 78 and the IETF Trust's Legal Provisions Relating to IETF Documents

(http://trustee.ietf.org/license-info) in effect on the date of publication of this document. Please review these documents carefully, as they describe your rights and restrictions with respect to this document. Code Components extracted from this document must include Simplified BSD License text as described in section $4 . e$ of the Trust Legal Provisions and are provided without warranty as described in the Simplified BSD License. 
Table of Contents

1. Introduction . . . . . . . . . . . . . . . . . . . . . . . . 3

1.1. Notational Conventions . • • • • . . . . . . • . . . . 3

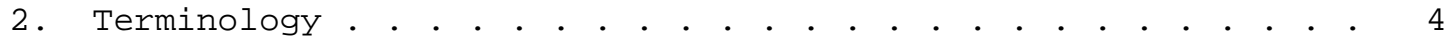

3. Example JWK . . . . . . . . . . . . . . . . . . . . . 5

4. JSON Web Key (JWK) Eormat . . . . . . . . . . . . . . . . 5

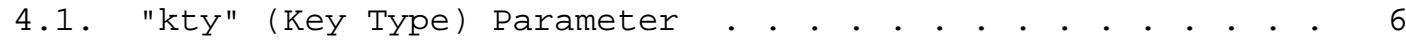

4.2. "use" (Public Key Use) Parameter . . . . . . . . . . . . . 6

4.3. "key_ops" (Key Operations) Parameter . . . . . . . . . . 7

4.4. "alg" (Algorithm) Parameter . . . . . . . . . . . . . . . 8

4.5. "kid" (Key ID) Parameter . . . . . . . . . . . . . . . . . 8

4.6. "x5u" (X.509 URL) Parameter . . . . . . . . . . . . . . . 8

4.7. "x5c" (X.509 Certificate Chain) Parameter . . . . . . . . 9

4.8. "x5t" (X.509 Certificate SHA-1 Thumbprint) Parameter . . 9

4.9. "x5t\#S256" (X.509 Certificate SHA-256 Thumbprint)

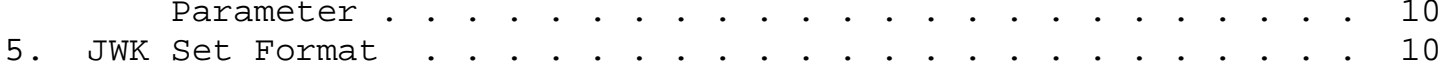

5.1. "keys" Parameter . . . . . . . . . . . . . . . . . . . . 10

6. String Comparison Rules . . . . . . . . . . . . . . . . . . 11

7. Encrypted JWK and Encrypted JWK Set Formats . . • • • • • . 11

8. IANA Considerations . . . . . . • . . . . . . . . . . . . . 11

8.1. JSON Web Key Parameters Registry . . . . . . . . . . . . 12

8.1.1. Registration Template . • . • • . • • • • • . . 12

8.1.2. Initial Registry Contents . . . . . . . . . . . . 13

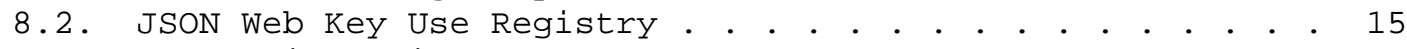

8.2.1. Registration Template. . . . . . . . . . . . . 15

8.2.2. Initial Registry Contents . . . . . . . . . . . 15

8.3. JSON Web Key Operations Registry • • • . . . . . . • • 16

8.3.1. Registration Template . . . . . . . . . . . . . . . . 16

8.3.2. Initial Registry Contents . . . . . . . . . . . 16

8.4. JSON Web Key Set Parameters Registry . . . . . . . . . 17

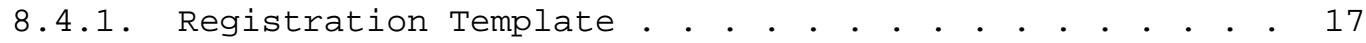

8.4.2. Initial Registry Contents . . . . . . . . . . . . . 18

8.5. Media Type Registration . . . . . . . . . . . . . . . 18

8.5.1. Registry Contents . . . . . . . . . . . . . . 18

9. Security Considerations . . . . . . . . . . . . . . . . . 19

9.1. Key Provenance and Trust . . . . . . . . . . . . . . . . 20

9.2. Preventing Disclosure of Non-public Key Information . . 20

9.3. RSA Private Key Representations and Blinding . . . . . . 21

9.4. Key Entropy and Random Values . . . . . . . . . . . . . . 21

10. References . . . . . . . . . . . . . . . . . . . . . . 21

10.1. Normative References . . . . . . . . . . . . . . . 21

10.2. Informative References . . . . . . . . . . . . . . . . 23

Appendix A. Example JSON Web Key Sets . . . . . . . . . . 25

A.1. Example Public Keys . . . . . . . . . . . . . . . . . . . 25

A.2. Example Private Keys . . . . . . . . . . . . . . . 25

A.3. Example Symmetric Keys . . . . . . . . . . . . . . . 27 


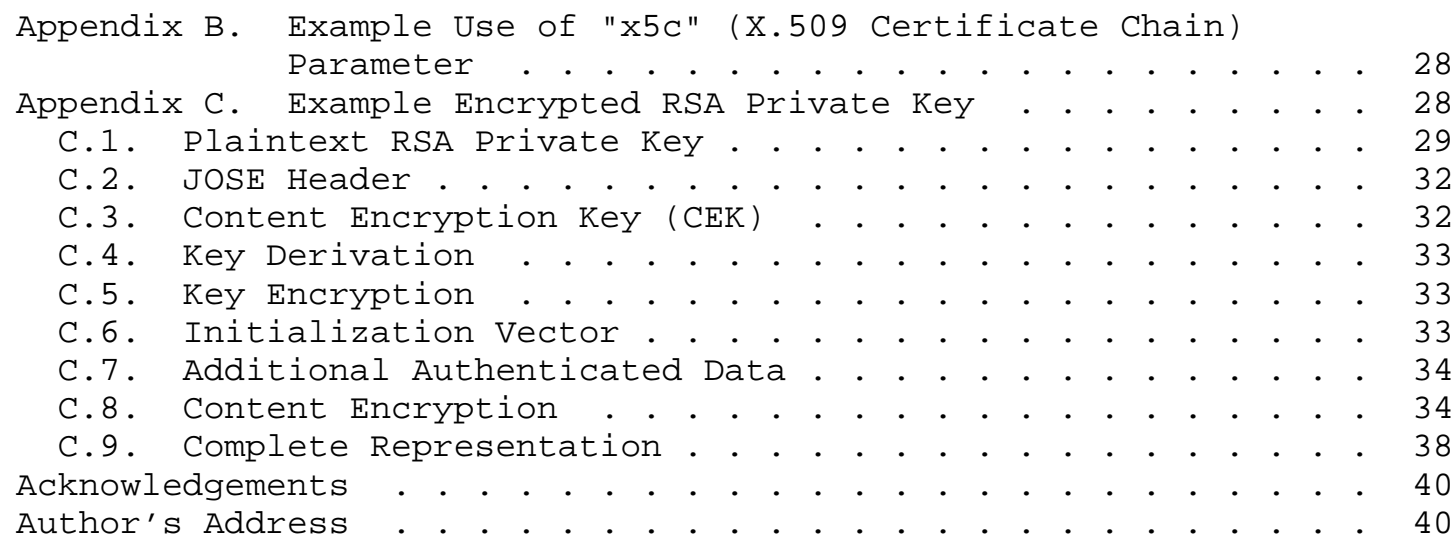

1. Introduction

A JSON Web Key (JWK) is a JavaScript Object Notation (JSON) [RFC7159] data structure that represents a cryptographic key. This specification also defines a JWK set JSON data structure that represents a set of JWKs. Cryptographic algorithms and identifiers for use with this specification are described in the separate JSON Web Algorithms (JWA) [JWA] specification and IANA registries established by that specification.

Goals for this specification do not include representing new kinds of certificate chains, representing new kinds of certified keys, or replacing X.509 certificates.

JWKs and JWK Sets are used in the JSON Web Signature [JWS] and JSON Web Encryption [JWE] specifications.

Names defined by this specification are short because a core goal is for the resulting representations to be compact.

\subsection{Notational Conventions}

The key words "MUST", "MUST NOT", "REQUIRED", "SHALL", "SHALL NOT", "SHOULD", "SHOULD NOT", "RECOMMENDED", "NOT RECOMMENDED", "MAY", and "OPTIONAL" in this document are to be interpreted as described in "Key words for use in RFCs to Indicate Requirement Levels" [RFC2119]. The interpretation should only be applied when the terms appear in all capital letters.

BASE64URL (OCTETS) denotes the base64url encoding of OCTETS, per Section 2 of [JWS]. 
UTF8 (STRING) denotes the octets of the UTF-8 [RFC3629] representation of STRING, where STRING is a sequence of zero or more Unicode [UNICODE] characters.

ASCII(STRING) denotes the octets of the ASCII [RFC20] representation of STRING, where STRING is a sequence of zero or more ASCII characters.

The concatenation of two values $A$ and $B$ is denoted as $A|| B$.

2. Terminology

The terms "JSON Web Signature (JWS)", "Base64url Encoding", "Collision-Resistant Name", "Header Parameter", and "JOSE Header" are defined by the JWS specification [JWS].

The terms "JSON Web Encryption (JWE)", "Additional Authenticated Data (AAD) ", "JWE Authentication Tag", "JWE Ciphertext", "JWE Compact Serialization", "JWE Encrypted Key", "JWE Initialization Vector", and "JWE Protected Header" are defined by the JWE specification [JWE] .

The terms "Ciphertext", "Digital Signature", "Message Authentication Code (MAC)", and "Plaintext" are defined by the "Internet Security Glossary, Version 2" [RFC4949].

These terms are defined by this specification:

JSON Web Key (JWK)

A JSON object that represents a cryptographic key. The members of the object represent properties of the key, including its value.

JWK Set

A JSON object that represents a set of JWKs. The JSON object MUST have a "keys" member, which is an array of JWKs. 


\section{Example JWK}

This section provides an example of a JWK. The following example JWK declares that the key is an Elliptic Curve [DSS] key, it is used with the P-256 Elliptic Curve, and its $x$ and $y$ coordinates are the base64url-encoded values shown. A key identifier is also provided for the key.

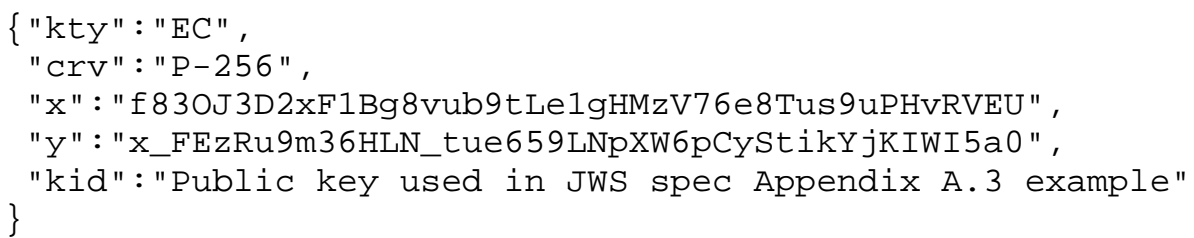

Additional example JWK values can be found in Appendix A.

4. JSON Web Key (JWK) Format

A JWK is a JSON object that represents a cryptographic key. The members of the object represent properties of the key, including its value. This JSON object MAY contain whitespace and/or line breaks before or after any JSON values or structural characters, in accordance with section 2 of RFC 7159 [RFC7159]. This document defines the key parameters that are not algorithm specific and, thus, common to many keys.

In addition to the common parameters, each JWK will have members that are key type specific. These members represent the parameters of the key. Section 6 of the JSON Web Algorithms (JWA) [JWA] specification defines multiple kinds of cryptographic keys and their associated members.

The member names within a JWK MUST be unique; JWK parsers MUST either reject JWKs with duplicate member names or use a JSON parser that returns only the lexically last duplicate member name, as specified in Section 15.12 (The JSON Object) of ECMAScript 5.1 [ECMAScript].

Additional members can be present in the JWK; if not understood by implementations encountering them, they MUST be ignored. Member names used for representing key parameters for different keys types need not be distinct. Any new member name should either be registered in the IANA "JSON Web Key Parameters" registry established by Section 8.1 or be a value that contains a Collision-Resistant Name. 


\section{1. "kty" (Key Type) Parameter}

The "kty" (key type) parameter identifies the cryptographic algorithm family used with the key, such as "RSA" or "EC". "kty" values should either be registered in the IANA "JSON Web Key Types" registry established by [JWA] or be a value that contains a collisionResistant Name. The "kty" value is a case-sensitive string. This member MUST be present in a JWK.

A list of defined "kty" values can be found in the IANA "JSON Web Key Types" registry established by [JWA]; the initial contents of this registry are the values defined in section 6.1 of [JWA] .

The key type definitions include specification of the members to be used for those key types. Members used with specific "kty" values can be found in the IANA "JSON Web Key Parameters" registry established by Section 8.1 .

4.2. "use" (Public Key Use) Parameter

The "use" (public key use) parameter identifies the intended use of the public key. The "use" parameter is employed to indicate whether a public key is used for encrypting data or verifying the signature on data.

Values defined by this specification are:

o "sig" (signature)

o "enc" (encryption)

Other values MAY be used. The "use" value is a case-sensitive string. Use of the "use" member is OPTIONAL, unless the application requires its presence.

When a key is used to wrap another key and a public key use designation for the first key is desired, the "enc" (encryption) key use value is used, since key wrapping is a kind of encryption. The "enc" value is also to be used for public keys used for key agreement operations.

Additional "use" (public key use) values can be registered in the IANA "JSON Web Key Use" registry established by Section 8.2. Registering any extension values used is highly recommended when this specification is used in open environments, in which multiple organizations need to have a common understanding of any extensions used. However, unregistered extension values can be used in closed environments, in which the producing and consuming organization will always be the same. 


\section{3. "key_ops" (Key Operations) Parameter}

The "key_ops" (key operations) parameter identifies the operation(s) for which the key is intended to be used. The "key_ops" parameter is intended for use cases in which public, private, or symmetric keys may be present.

Its value is an array of key operation values. Values defined by this specification are:

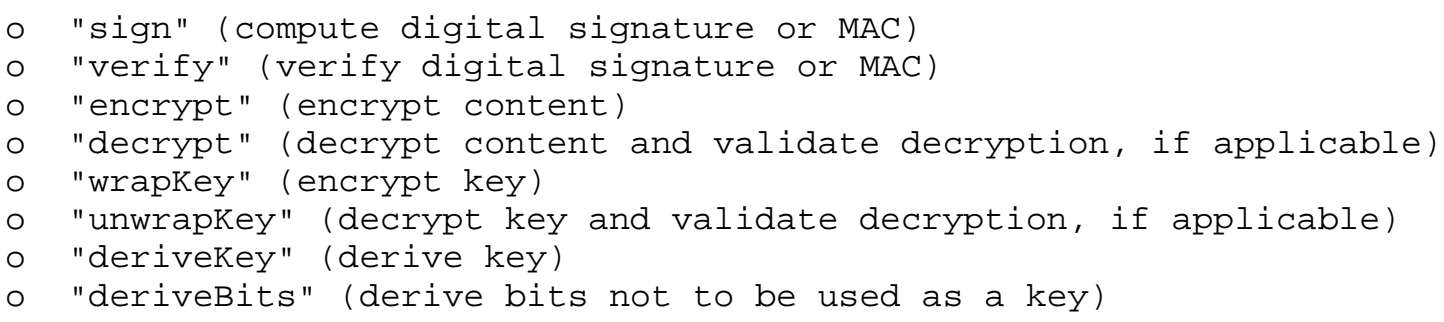

(Note that the "key_ops" values intentionally match the "KeyUsage" values defined in the Web Cryptography API

[W3C.CR-WebCryptoAPI-20141211] specification.)

Other values MAY be used. The key operation values are casesensitive strings. Duplicate key operation values MUST NOT be present in the array. Use of the "key_ops" member is OPTIONAL, unless the application requires its presence.

Multiple unrelated key operations SHOULD NOT be specified for a key because of the potential vulnerabilities associated with using the same key with multiple algorithms. Thus, the combinations "sign" with "verify", "encrypt" with "decrypt", and "wrapkey" with "unwrapkey" are permitted, but other combinations SHOULD NOT be used.

Additional "key_ops" (key operations) values can be registered in the IANA "JSON Web Key Operations" registry established by Section 8.3. The same considerations about registering extension values apply to the "key_ops" member as do for the "use" member.

The "use" and "key_ops" JWK members SHOULD NOT be used together; however, if both are used, the information they convey MUST be consistent. Applications should specify which of these members they use, if either is to be used by the application. 


\section{4. "alg" (Algorithm) Parameter}

The "alg" (algorithm) parameter identifies the algorithm intended for use with the key. The values used should either be registered in the IANA "JSON Web Signature and Encryption Algorithms" registry established by [JWA] or be a value that contains a CollisionResistant Name. The "alg" value is a case-sensitive ASCII string. Use of this member is OPTIONAL.

4.5. "kid" (Key ID) Parameter

The "kid" (key ID) parameter is used to match a specific key. This is used, for instance, to choose among a set of keys within a JWK set during key rollover. The structure of the "kid" value is unspecified. When "kid" values are used within a JWK Set, different keys within the JWK set SHOULD use distinct "kid" values. (One example in which different keys might use the same "kid" value is if they have different "kty" (key type) values but are considered to be equivalent alternatives by the application using them.) The "kid" value is a case-sensitive string. Use of this member is OPTIONAL. When used with JWS or JWE, the "kid" value is used to match a JWS or JWE "kid" Header Parameter value.

\section{6. "x5u" (X.509 URL) Parameter}

The "x5u" (X.509 URL) parameter is a URI [RFC3986] that refers to a resource for an X.509 public key certificate or certificate chain [RFC5280]. The identified resource MUST provide a representation of the certificate or certificate chain that conforms to RFC 5280 [RFC5280] in PEM-encoded form, with each certificate delimited as specified in section 6.1 of RFC 4945 [RFC4945]. The key in the first certificate MUST match the public key represented by other members of the JWK. The protocol used to acquire the resource MUST provide integrity protection; an HTTP GET request to retrieve the certificate MUST use TLS [RFC2818] [RFC5246]; the identity of the server MUST be validated, as per section 6 of RFC 6125 [RFC6125]. Use of this member is OPTIONAL.

While there is no requirement that optional JWK members providing key usage, algorithm, or other information be present when the "x5u" member is used, doing so may improve interoperability for applications that do not handle PKIX certificates [RFC5280]. If other members are present, the contents of those members MUST be semantically consistent with the related fields in the first certificate. For instance, if the "use" member is present, then it MUST correspond to the usage that is specified in the certificate, 
when it includes this information. Similarly, if the "alg" member is present, it MUST correspond to the algorithm specified in the certificate.

\section{7. "x5c" (X.509 Certificate Chain) Parameter}

The "x5c" (X.509 certificate chain) parameter contains a chain of one or more PKIX certificates [RFC5280]. The certificate chain is represented as a JSON array of certificate value strings. Each string in the array is a base64-encoded (Section 4 of [RFC4648] -not base64url-encoded) DER [ITU.X690.1994] PKIX certificate value. The PKIX certificate containing the key value MUST be the first certificate. This MAY be followed by additional certificates, with each subsequent certificate being the one used to certify the previous one. The key in the first certificate MUST match the public key represented by other members of the JWK. Use of this member is OPTIONAL.

As with the "x5u" member, optional JWK members providing key usage, algorithm, or other information MAY also be present when the "x 5 " member is used. If other members are present, the contents of those members MUST be semantically consistent with the related fields in the first certificate. See the last paragraph of section 4.6 for additional guidance on this.

\section{8. "x5t" (X.509 Certificate SHA-1 Thumbprint) Parameter}

The "x5t" (X.509 certificate SHA-1 thumbprint) parameter is a base64url-encoded SHA-1 thumbprint (a.k.a. digest) of the DER encoding of an X.509 certificate [RFC5280]. Note that certificate thumbprints are also sometimes known as certificate fingerprints. The key in the certificate MUST match the public key represented by other members of the JWK. Use of this member is OPTIONAL.

As with the "x5u" member, optional JWK members providing key usage, algorithm, or other information MAY also be present when the "x5t" member is used. If other members are present, the contents of those members MUST be semantically consistent with the related fields in the referenced certificate. See the last paragraph of Section 4.6 for additional guidance on this. 
4.9. "x5t\#S256" (X.509 Certificate SHA-256 Thumbprint) Parameter

The "x5t\#S256" (X.509 certificate SHA-256 thumbprint) parameter is a base64url-encoded SHA-256 thumbprint (a.k.a. digest) of the DER encoding of an X.509 certificate [RFC5280]. Note that certificate thumbrints are also sometimes known as certificate fingerprints. The key in the certificate MUST match the public key represented by other members of the JWK. Use of this member is OPTIONAL.

As with the "x5u" member, optional JWK members providing key usage, algorithm, or other information MAY also be present when the "x5t\#S256" member is used. If other members are present, the contents of those members MUST be semantically consistent with the related fields in the referenced certificate. See the last paragraph of Section 4.6 for additional guidance on this.

5. JWK Set Format

A JWK set is a JSON object that represents a set of JWKs. The JSON object MUST have a "keys" member, with its value being an array of JWKs. This JSON object MAY contain whitespace and/or line breaks.

The member names within a JWK Set MUST be unique; JWK Set parsers MUST either reject JWK Sets with duplicate member names or use a JSON parser that returns only the lexically last duplicate member name, as specified in Section 15.12 ("The JSON Object") of ECMAScript 5.1 [ECMAScript].

Additional members can be present in the JWK Set; if not understood by implementations encountering them, they MUST be ignored.

Parameters for representing additional properties of JWK Sets should either be registered in the IANA "JSON Web Key Set Parameters" registry established by section 8.4 or be a value that contains a Collision-Resistant Name.

Implementations SHOULD ignore JWKs within a JWK Set that use "kty" (key type) values that are not understood by them, that are missing required members, or for which values are out of the supported ranges.

\section{1. "keys" Parameter}

The value of the "keys" parameter is an array of JWK values. By default, the order of the JWK values within the array does not imply an order of preference among them, although applications of JWK sets can choose to assign a meaning to the order for their purposes, if desired. 
6. String Comparison Rules

The string comparison rules for this specification are the same as those defined in section 5.3 of [JWS].

7. Encrypted JWK and Encrypted JWK Set Formats

Access to JWKs containing non-public key material by parties without legitimate access to the non-public information MUST be prevented. This can be accomplished by encrypting the JWK when potentially observable by such parties to prevent the disclosure of private or symmetric key values. The use of an Encrypted JWK, which is a JWE with the UTF-8 encoding of a JWK as its plaintext value, is recommended for this purpose. The processing of Encrypted JWKs is identical to the processing of other JWEs. A "cty" (content type) Header Parameter value of "jwk+json" MUST be used to indicate that the content of the JWE is a JWK, unless the application knows that the encrypted content is a JWK by another means or convention, in which case the "cty" value would typically be omitted.

JWK Sets containing non-public key material will also need to be encrypted under these circumstances. The use of an Encrypted JWK Set, which is a JWE with the UTF-8 encoding of a JWK set as its plaintext value, is recommended for this purpose. The processing of Encrypted JWK Sets is identical to the processing of other JWEs. A "cty" (content type) Header Parameter value of "jwk-set+json" MUST be used to indicate that the content of the JWE is a JWK set, unless the application knows that the encrypted content is a JWK set by another means or convention, in which case the "cty" value would typically be omitted.

See Appendix C for an example encrypted JWK.

\section{IANA Considerations}

The following registration procedure is used for all the registries established by this specification.

The registration procedure for values is Specification Required [RFC5226] after a three-week review period on the jose-reg-reviewlietf.org mailing list, on the advice of one or more Designated Experts. However, to allow for the allocation of values prior to publication, the Designated Experts may approve registration once they are satisfied that such a specification will be published.

Registration requests sent to the mailing list for review should use an appropriate subject (e.g., "Request to register JWK parameter: example"). 
Within the review period, the Designated Experts will either approve or deny the registration request, communicating this decision to the review list and IANA. Denials should include an explanation and, if applicable, suggestions as to how to make the request successful. Registration requests that are undetermined for a period longer than 21 days can be brought to the IESG's attention (using the iesgeietf.org mailing list) for resolution.

Criteria that should be applied by the Designated Experts include determining whether the proposed registration duplicates existing functionality, whether it is likely to be of general applicability or useful only for a single application, and whether the registration description is clear.

IANA must only accept registry updates from the Designated Experts and should direct all requests for registration to the review mailing list.

It is suggested that multiple Designated Experts be appointed who are able to represent the perspectives of different applications using this specification, in order to enable broadly informed review of registration decisions. In cases where a registration decision could be perceived as creating a conflict of interest for a particular Expert, that Expert should defer to the judgment of the other Experts.

\subsection{JSON Web Key Parameters Registry}

This section establishes the IANA "JSON Web Key Parameters" registry for JWK parameter names. The registry records the parameter name, the key type(s) that the parameter is used with, and a reference to the specification that defines it. It also records whether the parameter conveys public or private information. This section registers the parameter names defined in section 4. The same JWK parameter name may be registered multiple times, provided that duplicate parameter registrations are only for key-type-specific JWK parameters; in this case, the meaning of the duplicate parameter name is disambiguated by the "kty" value of the JWK containing it.

8.1.1. Registration Template

Parameter Name:

The name requested (e.g., "kid"). Because a core goal of this specification is for the resulting representations to be compact, it is RECOMMENDED that the name be short -- not to exceed 8 characters without a compelling reason to do so. This name is case sensitive. Names may not match other registered names in a case-insensitive manner unless the Designated Experts state that 
there is a compelling reason to allow an exception. However, matching names may be registered, provided that the accompanying sets of "kty" values that the parameter name is used with are disjoint; for the purposes of matching "kty" values, "*" matches all values.

Parameter Description:

Brief description of the parameter (e.g., "Key ID") .

Used with "kty" Value(s):

The key type parameter value(s) that the parameter name is to be used with, or the value "*" if the parameter value is used with all key types. Values may not match other registered "kty" values in a case-insensitive manner when the registered parameter name is the same (including when the parameter name matches in a caseinsensitive manner) unless the Designated Experts state that there is a compelling reason to allow an exception.

Parameter Information Class:

Registers whether the parameter conveys public or private information. Its value must be either Public or Private.

Change Controller:

For Standards Track RFCs, list the "IESG". For others, give the name of the responsible party. Other details (e.g., postal address, email address, home page URI) may also be included.

Specification Document (s):

Reference to the document or documents that specify the parameter, preferably including URIs that can be used to retrieve copies of the documents. An indication of the relevant sections may also be included but is not required.

\subsubsection{Initial Registry Contents}

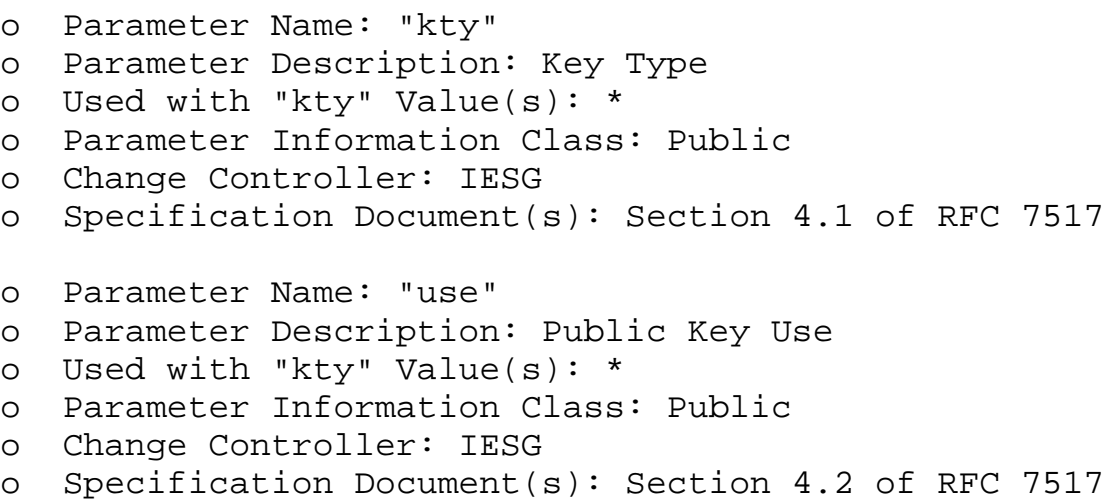




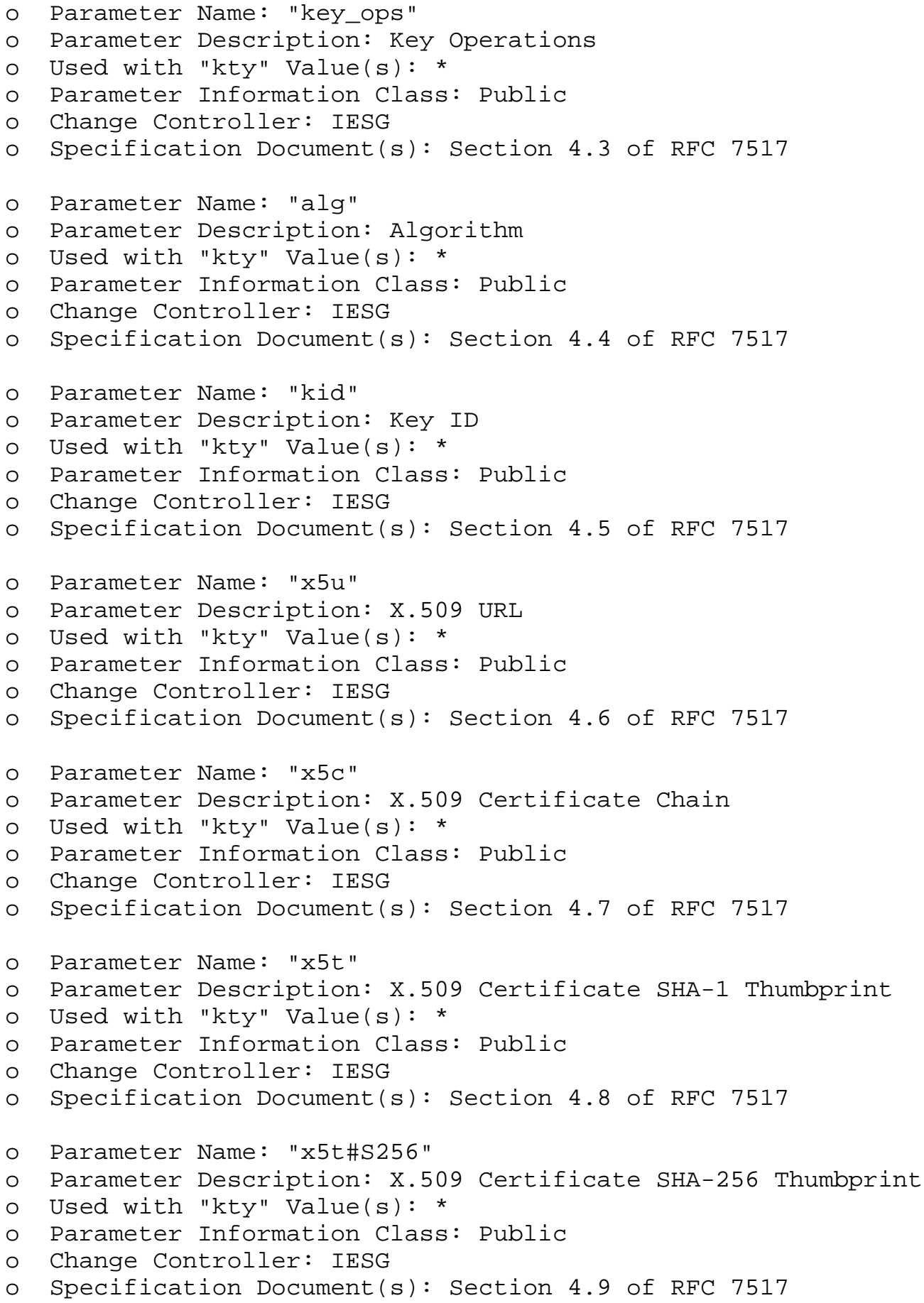




\subsection{JSON Web Key Use Registry}

This section establishes the IANA "JSON Web Key Use" registry for JWK "use" (public key use) member values. The registry records the public key use value and a reference to the specification that defines it. This section registers the parameter names defined in Section 4.2 .

\subsubsection{Registration Template}

Use Member Value:

The name requested (e.g., "sig"). Because a core goal of this specification is for the resulting representations to be compact, it is RECOMMENDED that the name be short -- not to exceed 8 characters without a compelling reason to do so. This name is case sensitive. Names may not match other registered names in a case-insensitive manner unless the Designated Experts state that there is a compelling reason to allow an exception.

Use Description: Brief description of the use (e.g., "Digital Signature or MAC").

Change Controller:

For Standards Track RFCs, list the "IESG". For others, give the name of the responsible party. Other details (e.g., postal address, email address, home page URI) may also be included.

Specification Document (s):

Reference to the document or documents that specify the parameter, preferably including URIs that can be used to retrieve copies of the documents. An indication of the relevant sections may also be included but is not required.

\subsubsection{Initial Registry Contents}

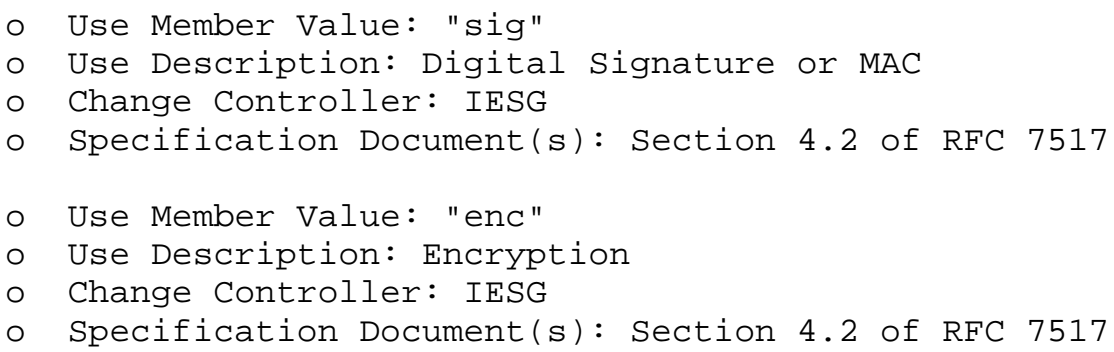




\subsection{JSON Web Key Operations Registry}

This section establishes the IANA "JSON Web Key Operations" registry for values of JWK "key_ops" array elements. The registry records the key operation value and a reference to the specification that defines it. This section registers the parameter names defined in Section 4.3 .

\subsubsection{Registration Template}

Key Operation Value:

The name requested (e.g., "sign"). Because a core goal of this specification is for the resulting representations to be compact, it is RECOMMENDED that the name be short -- not to exceed 8 characters without a compelling reason to do so. This name is case sensitive. Names may not match other registered names in a case-insensitive manner unless the Designated Experts state that there is a compelling reason to allow an exception.

Key Operation Description: Brief description of the key operation (e.g., "Compute digital signature or MAC").

Change Controller:

For Standards Track RFCs, list the "IESG". For others, give the name of the responsible party. Other details (e.g., postal address, email address, home page URI) may also be included.

Specification Document (s): Reference to the document or documents that specify the parameter, preferably including URIs that can be used to retrieve copies of the documents. An indication of the relevant sections may also be included but is not required.

\subsubsection{Initial Registry Contents}

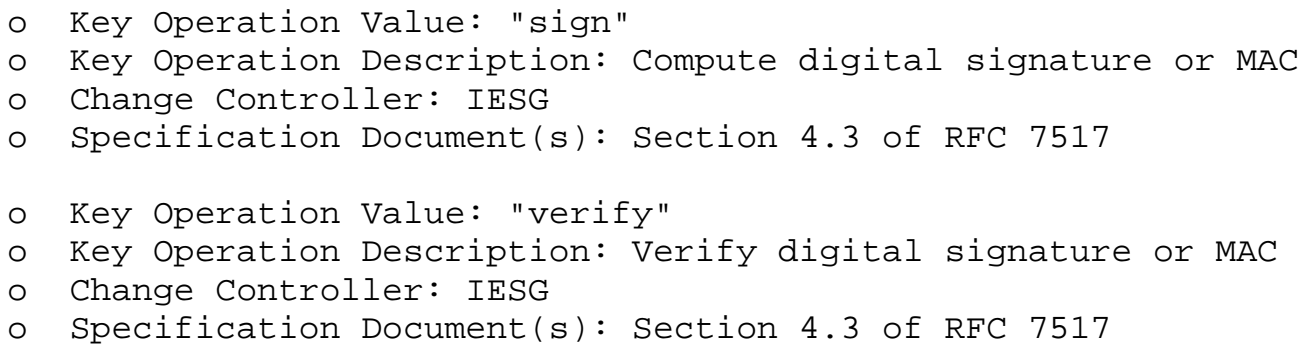


- Key Operation Value: "encrypt"

- Key Operation Description: Encrypt content

o Change Controller: IESG

o Specification Document(s): Section 4.3 of RFC 7517

- Key Operation Value: "decrypt"

- Key Operation Description: Decrypt content and validate decryption, if applicable

- Change Controller: IESG

- Specification Document(s) : Section 4.3 of RFC 7517

- Key Operation Value: "wrapkey"

- Key Operation Description: Encrypt key

- Change Controller: IESG

- Specification Document(s): Section 4.3 of RFC 7517

- Key Operation Value: "unwrapkey"

- Key Operation Description: Decrypt key and validate decryption, if applicable

- Change Controller: IESG

- Specification Document(s): Section 4.3 of RFC 7517

- Key Operation Value: "deriveKey"

- Key Operation Description: Derive key

- Change Controller: IESG

o Specification Document(s): Section 4.3 of RFC 7517

- Key Operation Value: "deriveBits"

- Key Operation Description: Derive bits not to be used as a key

o Change Controller: IESG

o Specification Document(s): Section 4.3 of RFC 7517

8.4. JSON Web Key Set Parameters Registry

This section establishes the IANA "JSON Web Key set Parameters" registry for JWK set parameter names. The registry records the parameter name and a reference to the specification that defines it. This section registers the parameter names defined in section 5.

8.4.1. Registration Template

Parameter Name:

The name requested (e.g., "keys"). Because a core goal of this specification is for the resulting representations to be compact, it is RECOMMENDED that the name be short -- not to exceed 8 characters without a compelling reason to do so. This name is case sensitive. Names may not match other registered names in a case-insensitive manner unless the Designated Experts state that there is a compelling reason to allow an exception. 


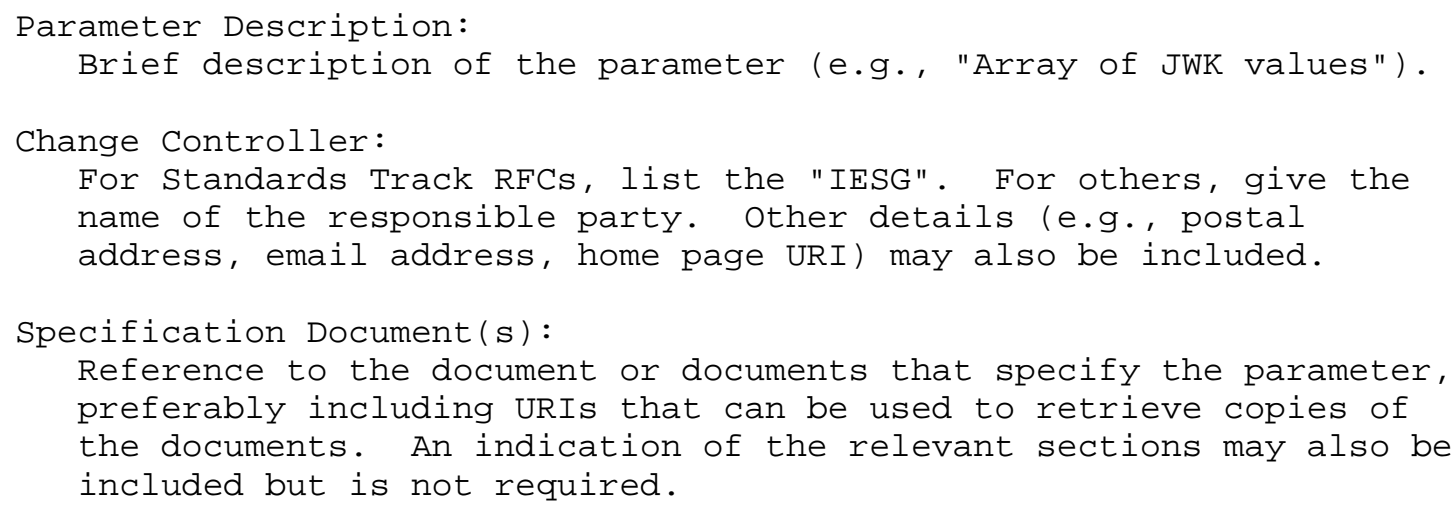

\subsubsection{Initial Registry Contents}

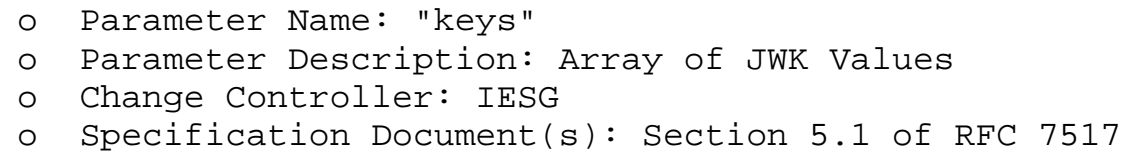

8.5. Media Type Registration

\subsubsection{Registry Contents}

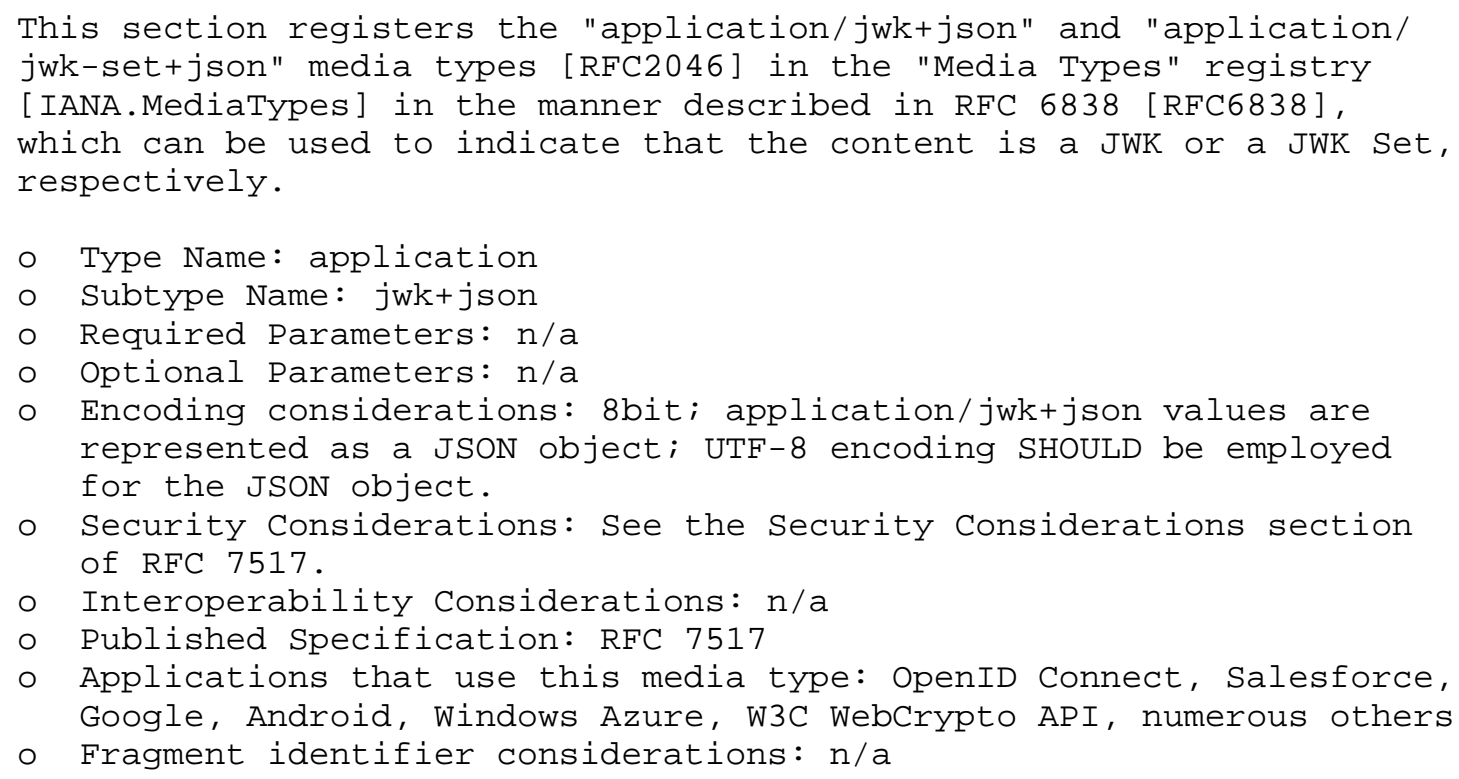


- Additional Information:

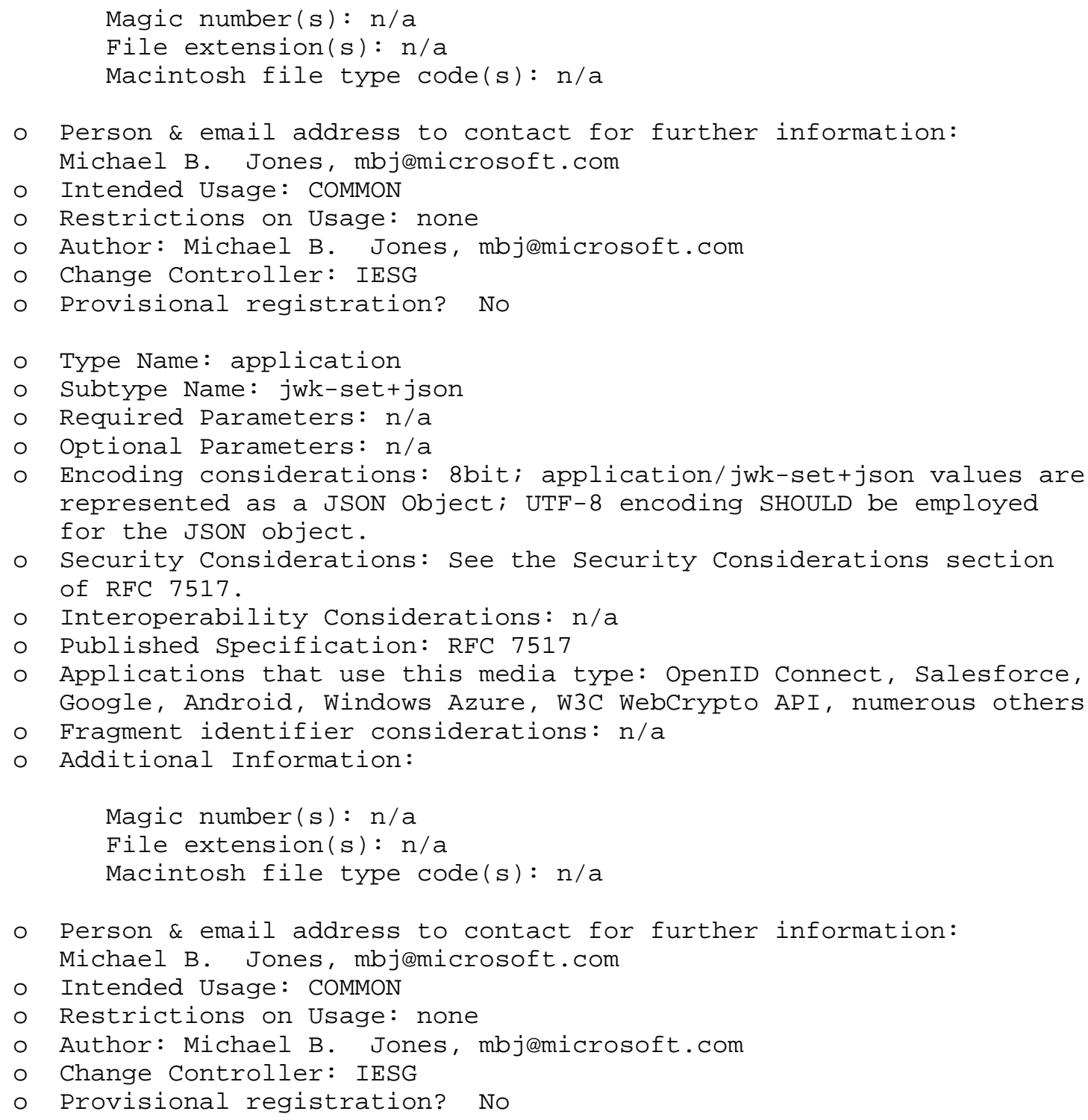

9. Security Considerations

All of the security issues that are pertinent to any cryptographic application must be addressed by JWS/JWE/JWK agents. Among these issues are protecting the user's asymmetric private and symmetric secret keys and employing countermeasures to various attacks. 


\subsection{Key Provenance and Trust}

One should place no more trust in the data cryptographically secured by a key than in the method by which it was obtained and in the trustworthiness of the entity asserting an association with the key. Any data associated with a key that is obtained in an untrusted manner should be treated with skepticism. See section 10.3 of [JWS] for security considerations on key origin authentication.

In almost all cases, applications make decisions about whether to trust a key based on attributes bound to the key, such as names, roles, and the key origin, rather than based on the key itself. When an application is deciding whether to trust a key, there are several ways that it can bind attributes to a JWK. Two example mechanisms are PKIX [RFC5280] and JSON Web Token (JWT) [JWT].

For instance, the creator of a JWK can include a PKIX certificate in the JWK's "x5c" member. If the application validates the certificate and verifies that the JWK corresponds to the subject public key in the certificate, then the JWK can be associated with the attributes in the certificate, such as the subject name, subject alternative names, extended key usages, and its signature chain.

As another example, a JWT can be used to associate attributes with a JWK by referencing the JWK as a claim in the JWT. The JWK can be included directly as a claim value or the JWT can include a TLSsecured URI from which to retrieve the JWK value. Either way, an application that gets a JWK via a JWT claim can associate it with the JWT's cryptographic properties and use these and possibly additional claims in deciding whether to trust the key.

The security considerations in section 12.3 of XML DSIG 2.0 [W3C.NOTE-xmldsig-core2-20130411] about the strength of a digital signature depending upon all the links in the security chain also apply to this specification.

The TLS Requirements in Section 8 of [JWS] also apply to this specification, except that the "x5u" JWK member is the only feature defined by this specification using TLS.

\subsection{Preventing Disclosure of Non-public Key Information}

Private and symmetric keys MUST be protected from disclosure to unintended parties. One recommended means of doing so is to encrypt JWKs or JWK sets containing them by using the JWK or JWK set value as the plaintext of a JWE. Of course, this requires that there be a 
secure way to obtain the key used to encrypt the non-public key information to the intended party and a secure way for that party to obtain the corresponding decryption key.

The security considerations in RFC 3447 [RFC3447] and RFC 6030

[RFC6030] about protecting private and symmetric keys, key usage, and information leakage also apply to this specification.

\subsection{RSA Private Key Representations and Blinding}

The RSA Key blinding operation [Kocher], which is a defense against some timing attacks, requires all of the RSA key values "n", "e", and "d". However, some RSA private key representations do not include the public exponent "e", but only include the modulus "n" and the private exponent "d". This is true, for instance, of the Java RSAPrivatekeyspec API, which does not include the public exponent "e" as a parameter. So as to enable RSA key blinding, such representations should be avoided. For Java, the RSAPrivateCrtKeySpec API can be used instead. Section 8.2.2(i) of the "Handbook of Applied Cryptography" [HAC] discusses how to compute the remaining RSA private key parameters, if needed, using only "n", "e", and "d".

9.4. Key Entropy and Random Values

See Section 10.1 of [JWS] for security considerations on key entropy and random values.

10. References

10.1. Normative References

[ECMAScript ]

Ecma International, "ECMAScript Language Specification, 5.1 Edition", ECMA Standard 262, June 2011, <http://www.ecma-international.org/ecma-262/5.1/ ECMA-262 . pdf>.

[ IANA. Mediatypes ] Internet Assigned Numbers Authority (IANA), "Media Types", <http: / /www.iana.org/assignments/media-types>.

[ITU.X690.1994] International Telecommunications Union, "Information Technology - ASN.1 encoding rules: Specification of Basic Encoding Rules (BER), Canonical Encoding Rules (CER) and Distinguished Encoding Rules (DER)", ITU-T Recommendation X.690, 1994 . 
[JWA] Jones, M., "JSON Web Algorithms (JWA) ", RFC 7518 , DOI 10.17487/RFC7518, May 2015, <http: //www.rfc-editor.org/info/rfc7518>.

[JWE] Jones, M. and J. Hildebrand, "JSON Web Encryption (JWE) ", RFC 7516, DOI 10.17487/RFC7516, May 2015, <http://wWw.rfc-editor.org/info/rfc7516>.

[JWS ] Jones, M., Bradley, J., and N. Sakimura, "JSON Web Signature (JWS)", RFC 7515, DOI 10.17487/RFC7515, May 2015, <http://WWW.rfc-editor.org/info/rfc7515>.

[RFC20] Cerf, V., "ASCII format for Network Interchange", STD 80, RFC 20, DOI 10.17487/RFC0020, October 1969, <http://www.rfc-editor.org/info/rfc20>.

[RFC2046] Freed, N. and N. Borenstein, "Multipurpose Internet Mail Extensions (MIME) Part Two: Media Types", RFC 2046, DOI 10.17487/RFC2046, November 1996, <http://www.rfc-editor.org/info/rfc2046>.

[RFC2119] Bradner, S., "Key words for use in RFCs to Indicate Requirement Levels", BCP 14, RFC 2119, DOI 10.17487/RFC2119, March 1997, <http://www.rfc-editor.org/info/rfc2119>.

[RFC2818] Rescorla, E., "HTTP Over TLS", RFC 2818, DOI 10.17487/RFC2818, May 2000, <http://www.rfc-editor.org/info/rfc2818>.

[RFC3629] Yergeau, F., "UTF-8, a transformation format of ISO 10646", STD 63, RFC 3629, DOI 10.17487/RFC3629, November 2003, <http://wWw.rfc-editor.org/info/rfc3629>.

[RFC3986] Berners-Lee, T., Fielding, R., and L. Masinter, "Uniform Resource Identifier (URI): Generic Syntax", STD 66, RFC 3986, DOI 10.17487/RFC3986, January 2005, <http://www.rfc-editor.org/info/rfc3986>.

[RFC4648] Josefsson, S., "The Base16, Base32, and Base64 Data Encodings", RFC 4648, DOI 10.17487/RFC4648, October 2006, <http://www.rfc-editor.org/info/rfc4648>.

[RFC4945] Korver, B., "The Internet IP Security PKI Profile of IKEV1/ISAKMP, IKEv2, and PKIX", RFC 4945, DOI $10.17487 /$ RFC4945, August 2007, <http://www.rfc-editor.org/info/rfc4945>. 
[RFC4949] Shirey, R., "Internet Security Glossary, Version 2", FYI 36, RFC 4949, DOI 10.17487/RFC4949, August 2007, <http://www.rfc-editor.org/info/rfc4949>.

[RFC5246] Dierks, T. and E. Rescorla, "The Transport Layer Security (TLS) Protocol Version 1.2", RFC 5246, DOI 10.17487/RFC5246, August 2008, <http://www.rfc-editor.org/info/rfc5246>.

[RFC5280] Cooper, D., Santesson, S., Farrell, S., Boeyen, S., Housley, R., and W. Polk, "Internet X.509 Public Key Infrastructure Certificate and Certificate Revocation List (CRL) Profile", RFC 5280, DOI 10.17487/RFC5280, May 2008, <http://www.rfc-editor.org/info/rfc5280>.

[RFC6125] Saint-Andre, P. and J. Hodges, "Representation and Verification of Domain-Based Application Service Identity within Internet Public Key Infrastructure Using X.509 (PKIX) Certificates in the Context of Transport Layer Security (TLS)", RFC 6125, DOI 10.17487/RFC6125, March 2011, <http://wWw.rfc-editor.org/info/rfc6125>.

[RFC7159] Bray, T., Ed., "The JavaScript Object Notation (JSON) Data Interchange Format", RFC 7159, DOI 10.17487/RFC7159, March 2014, <http://wWw.rfc-editor.org/info/rfc7159>.

[UNICODE] The Unicode Consortium, "The Unicode Standard", <http://www. unicode.org/versions/latest/>.

\subsection{Informative References}

[DSS] National Institute of Standards and Technology (NIST), "Digital Signature Standard (DSS)", FIPS PUB 186-4, July 2013, <http://nvlpubs.nist.gov/nistpubs/FIPS/ NIST.FIPS.186-4.pdf>.

[HAC] Menezes, A., van Oorschot, P., and S. Vanstone, "Handbook of Applied Cryptography", CRC Press, October 1996, <http://cacr. uwaterloo.ca/hac/>.

[JWT] Jones, M., Bradley, J., and N. Sakimura, "JSON Web Token (JWT) ", RFC 7519, DOI 10.17487/RFC7519, May 2015, <http://www.rfc-editor.org/info/rfc7519>. 
[Kocher] Kocher, P., "Timing Attacks on Implementations of Diffe-Hellman, RSA, DSS, and Other Systems", In Proceedings of the 16th Annual International Cryptology Conference Advances in Cryptology, Springer-Verlag, pp. $104-113,1996$.

[MagicSignatures]

Panzer, J., Ed., Laurie, B., and D. Balfanz, "Magic Signatures", January 2011, <http://salmon-protocol.googlecode.com/svn/trunk/ draft-panzer-magicsig-01.html>.

[RFC3447] Jonsson, J. and B. Kaliski, "Public-Key Cryptography Standards (PKCS) \#1: RSA Cryptography specifications Version 2.1", RFC 3447, DOI 10.17487/RFC3447, February 2003, <http://wWw.rfc-editor.org/info/rfc3447>.

[RFC5226] Narten, T. and H. Alvestrand, "Guidelines for Writing an IANA Considerations Section in RFCs", BCP 26, RFC 5226, DOI 10.17487/RFC5226, May 2008, <http://www.rfc-editor.org/info/rfc5226>.

[RFC6030] Hoyer, P., Pei, M., and S. Machani, "Portable Symmetric Key Container (PSKC)", RFC 6030, DOI 10.17487/RFC6030, October 2010, <http://www.rfc-editor.org/info/rfc6030>.

[RFC6838] Freed, N., Klensin, J., and T. Hansen, "Media Type Specifications and Registration Procedures", BCP 13, RFC 6838, DOI 10.17487/RFC6838, January 2013, <http://www.rfc-editor.org/info/rfc6838>.

[W3C.CR-WebCryptoAP I-20141211]

Sleevi, R. and M. Watson, "Web Cryptography API", World Wide Web Consortium Candidate Recommendation CR-WebCryptoAPI-20141211, December 2014, <http: / /wWw.w3.org/TR/2014/CR-WebCryptoAPI-20141211/>.

[W3C.NOTE-xmldsig-core2-20130411]

Eastlake, D., Reagle, J., Solo, D., Hirsch, F., Roessler, T., Yiu, K., Datta, P., and S. Cantor, "XML Signature Syntax and Processing Version 2.0", World Wide Web Consortium Note NOTE-xmldsig-core2-20130411, April 2013, <http: //wWw.w3.org/TR/2013/NOTE-xmldsig-core2-20130411/>. 
Appendix A. Example JSON Web Key Sets

\section{A.1. Example Public Keys}

The following example JWK set contains two public keys represented as JWKs: one using an Elliptic Curve algorithm and a second one using an RSA algorithm. The first specifies that the key is to be used for encryption. The second specifies that the key is to be used with the "RS256" algorithm. Both provide a key ID for key matching purposes. In both cases, integers are represented using the base64url encoding of their big-endian representations. (Line breaks within values are for display purposes only.)

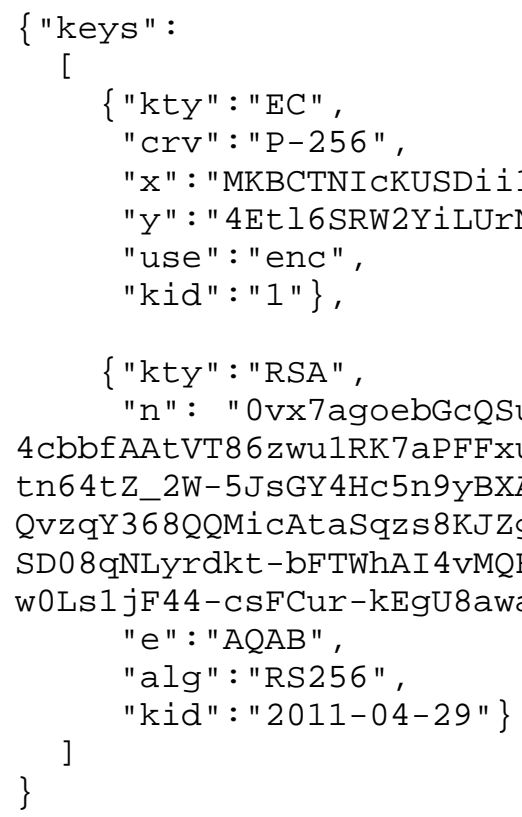

A.2. Example Private Keys

The following example JWK Set contains two keys represented as JWKs containing both public and private key values: one using an Elliptic Curve algorithm and a second one using an RSA algorithm. This example extends the example in the previous section, adding private key values. (Line breaks within values are for display purposes only.) 


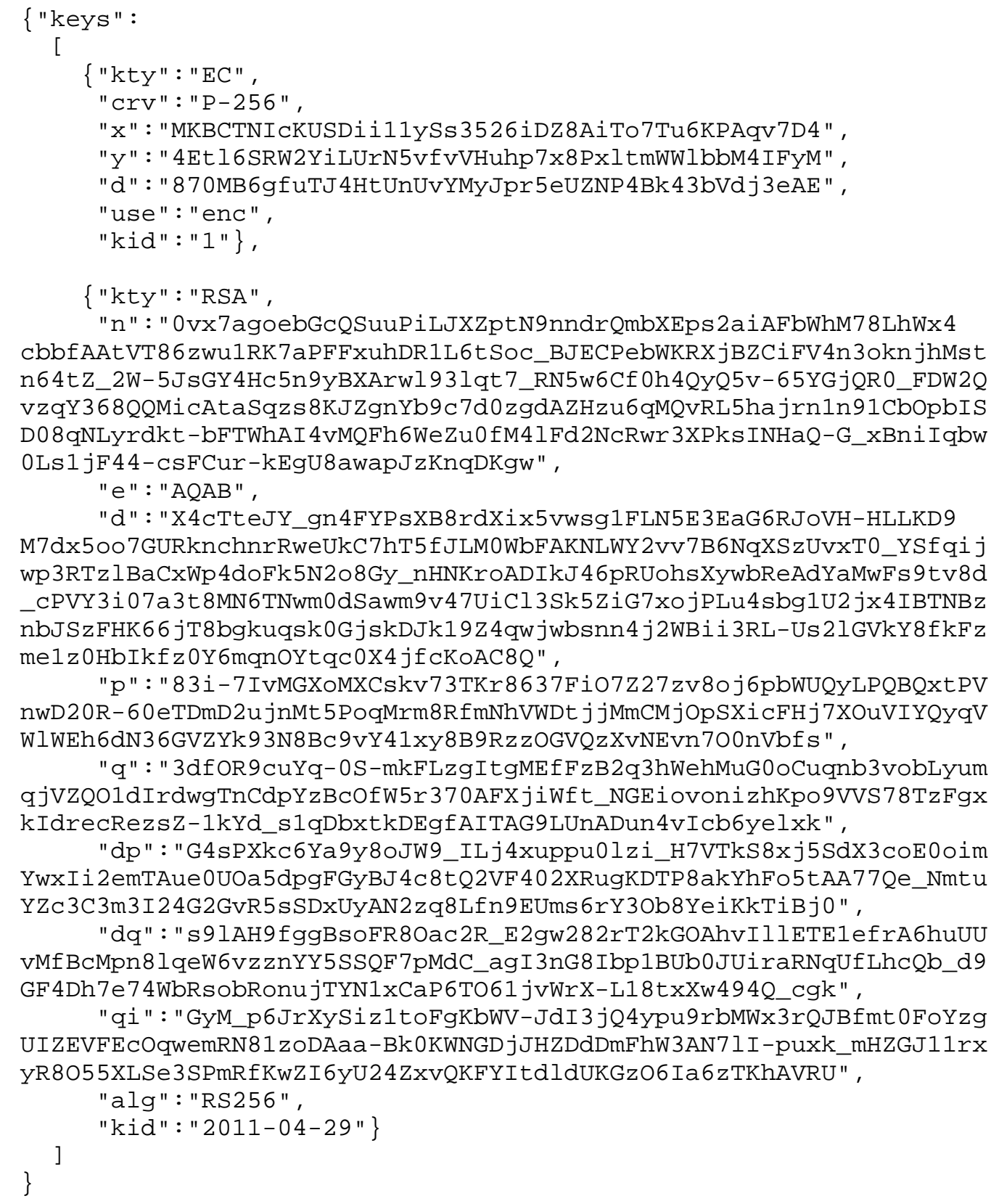




\section{A.3. Example Symmetric Keys}

The following example JWK set contains two symmetric keys represented as JWKs: one designated as being for use with the AES Key Wrap algorithm and a second one that is an HMAC key. (Line breaks within values are for display purposes only.)

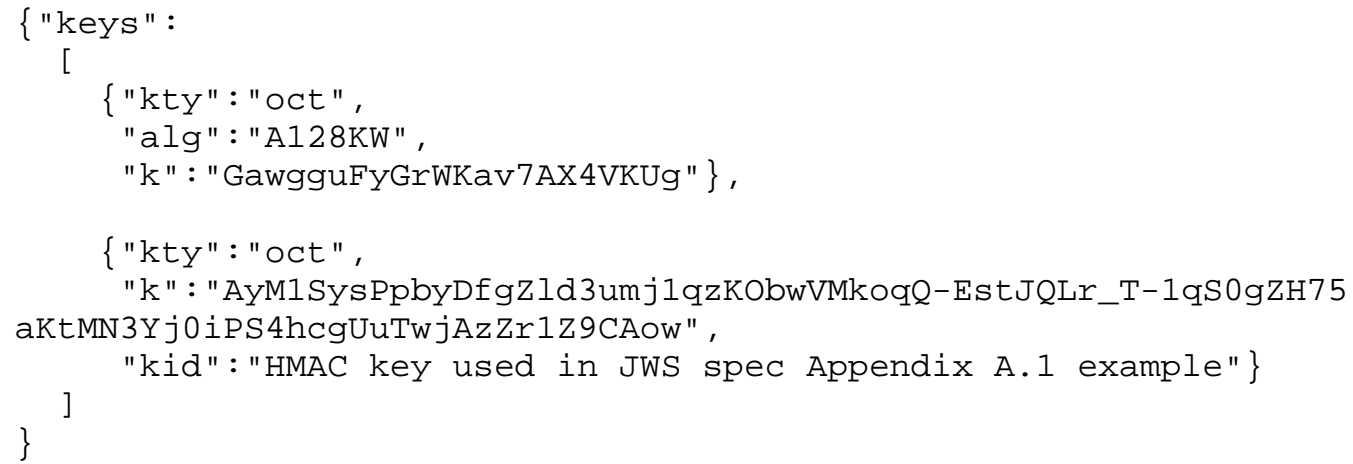


Appendix B. Example Use of "x5c" (X.509 Certificate Chain) Parameter

The following is an example of a JWK with a RSA signing key represented both as an RSA public key and as an X.509 certificate using the "x5c" parameter (with line breaks within values for display purposes only):

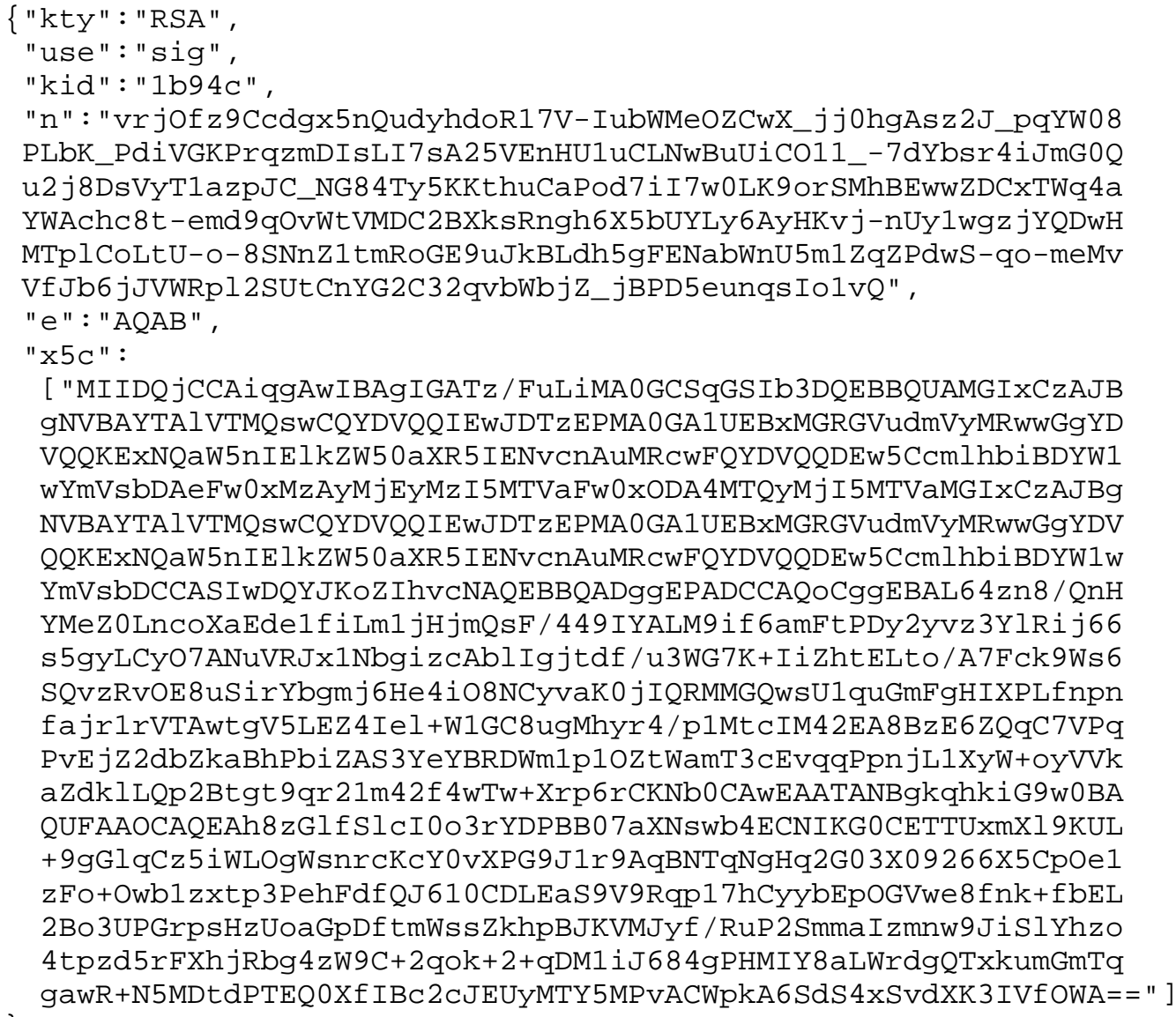

Appendix C. Example Encrypted RSA Private Key

This example encrypts an RSA private key to the recipient using "PBES2-HS256+A128KW" for key encryption and "A128CBC+HS256" for content encryption.

NOTE: Unless otherwise indicated, all line breaks are included solely for readability. 


\section{C.1. Plaintext RSA Private Key}

The following RSA key is the plaintext for the authenticated encryption operation, formatted as a JWK (with line breaks within values for display purposes only):

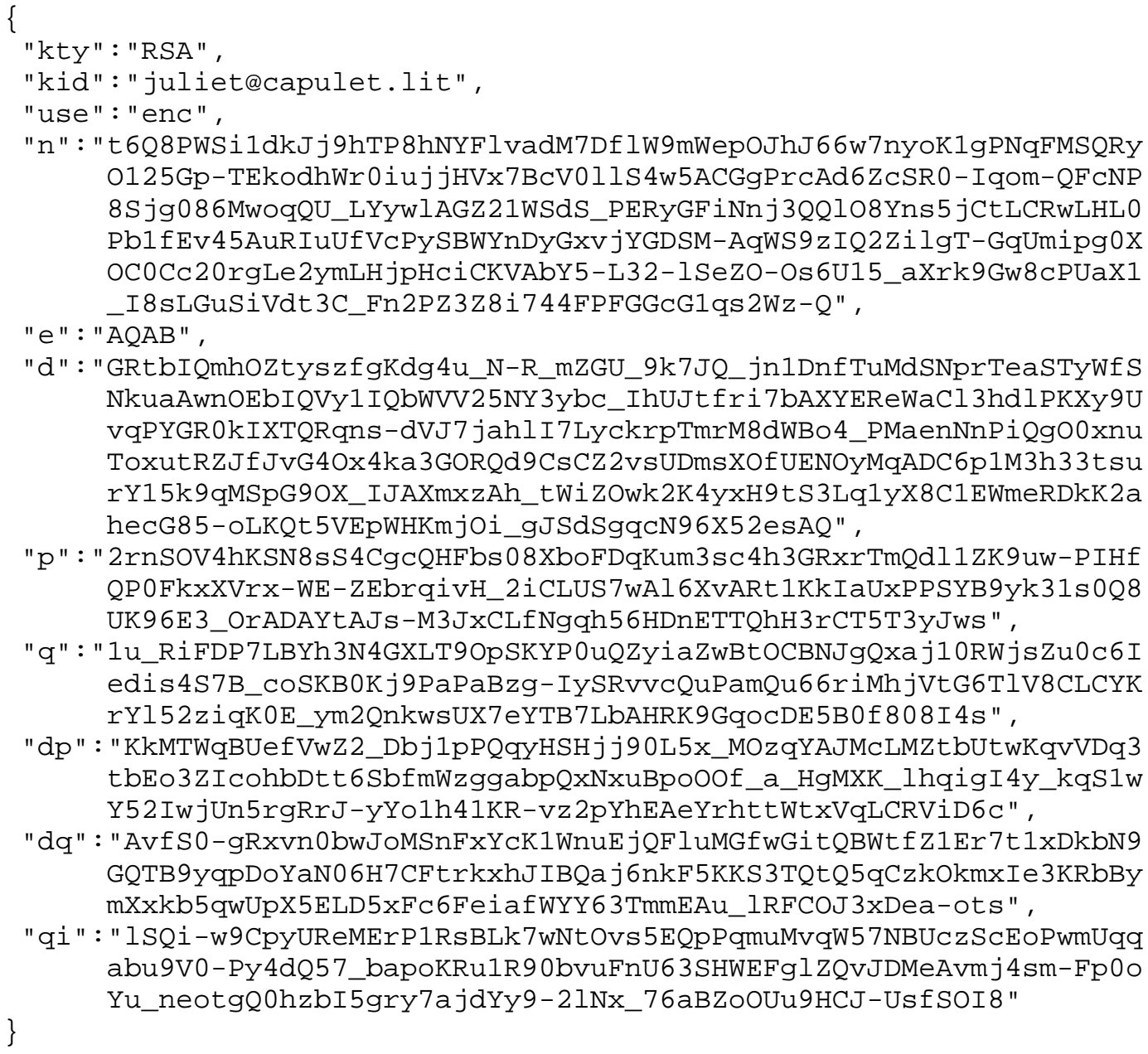
NkuaAwnOEbIQVy1IQbWVV2 5NY3ybc_IhUJtfri 7bAXYEReWaCl3hdlPKXy9U vqPYGR0kIXTQRqns-dVJ7 jahl I 7LyckrpTmrM8dWBo4_PMaenNnPiQgO0xnu ToxutRZJfJvG4 Ox 4 ka3GORQd9CsCZ2vsUDmsXOfUENOyMqADC6p1M3h33t su rY15k9qMSpG90X_IJAXmxzAh_tWizOwk2K4yxH9tS3Lq1yX8C1EWmeRDkK2a hecG85-oLKQt 5VEpWHKmjOi_gJSdSgqcN96X52esAQ",

"p" : "2rnSOV4hKSN8s S4 CgcQHFbs 0 8XboFDqKum3sc4h3GRxrTmQdl1ZK9uw-P IHf QP0FkxXVrx-WE-ZEbrqivH_2iCLUS7wAl6XvARt1KkIaUxPPSYB9yk31s0Q8 UK96E3_OrADAYtAJs-M3JxCLfNgqh56HDnETTQhH3rCT5T3yJws",

"q" : "1u_RiFDP7LBYh3N4GXLT 90pSKYP0uQZyiaZwBtOCBNJgQxaj10RWjsZu0c6I edis4S7B_coSKB0Kj9PaPaBzg-IySRvVCQuPamQu66riMh jVtG6TIV8CLCYK rYl52ziqK0E_ym2QnkwsUX7eYTB7LbAHRK9GqocDE5B0 f808I $4 \mathrm{~s}$ ",

"dp" : "KkMTWqBUefVwZ2_Dbj1pPQqyHSH j j90L5x_MOzqYAJMcLMZtbUtwKqvVDq3 tbEo3ZIcohbDtt6SbfmWzggabpQxNxuBpooof_a_HgMXK_lhqigI4y_kqS1w Y52IwjUn5rgRrJ-yYo1h41KR-vz2pYhEAeYrhttWtxVqLCRViD 6c",

"dq" : "AvfS0-gRxvn0bwJoMSnFxYcK1WnuEjQFluMGfwGitQBWtfZ1Er7t1xDkbN9 GQTB 9yqpDoYaN0 6H 7CFtrkxhJIBQa j6nkF5KKS3TQtQ5 qCzkOkmxIe3KRbBy mXxkb5qwUpX5ELD5xFc6FeiafWYY63TmmEAu_lRFCOJ3xDea-ots",

"qi " : "1SQi-w9CpyUReMErP1RsBLk 7wNt Ovs 5EQpP qmuMvqW57NBUcz S CEoP wmUqq abu 9V0-Py4dQ57_bapoKRu1R90bvuFnU63SHWEFglZQvJDMeAvmj4sm-Fp0o Yu_neotgQ0hzbI5gry7ajdYy9-2INx_76aBZoOUu9HCJ-UsfSOI8 "

The octets representing the plaintext used in this example (using JSON array notation) are:

$[123,34,107,116,121,34,58,34,82,83,65,34,44,34,107$, $105,100,34,58,34,106,117,108,105,101,116,64,99,97,112$, $117,108,101,116,46,108,105,116,34,44,34,117,115,101,34$, $58,34,101,110,99,34,44,34,110,34,58,34,116,54,81,56$, $80,87,83,105,49,100,107,74,106,57,104,84,80,56,104,78$, 
$89,70,108,118,97,100,77,55,68,102,108,87,57,109,87$, $101,112,79,74,104,74,54,54,119,55,110,121,111,75,49$, $103,80,78,113,70,77,83,81,82,121,79,49,50,53,71,112$, $45,84,69,107,111,100,104,87,114,48,105,117,106,106,72$, $86,120,55,66,99,86,48,108,108,83,52,119,53,65,67,71$, $103,80,114,99,65,100,54,90,99,83,82,48,45,73,113,111$, $109,45,81,70,99,78,80,56,83,106,103,48,56,54,77,119$, $111,113,81,85,95,76,89,121,119,108,65,71,90,50,49,87$, $83,100,83,95,80,69,82,121,71,70,105,78,110,106,51,81$, $81,108,79,56,89,110,115,53,106,67,116,76,67,82,119,76$, $72,76,48,80,98,49,102,69,118,52,53,65,117,82,73,117$, $85,102,86,99,80,121,83,66,87,89,110,68,121,71,120,118$, $106,89,71,68,83,77,45,65,113,87,83,57,122,73,81,50$, $90,105,108,103,84,45,71,113,85,109,105,112,103,48,88$, $79,67,48,67,99,50,48,114,103,76,101,50,121,109,76,72$, $106,112,72,99,105,67,75,86,65,98,89,53,45,76,51,50$, $45,108,83,101,90,79,45,79,115,54,85,49,53,95,97,88$, $114,107,57,71,119,56,99,80,85,97,88,49,95,73,56,115$, $76,71,117,83,105,86,100,116,51,67,95,70,110,50,80,90$, $51,90,56,105,55,52,52,70,80,70,71,71,99,71,49,113$, $115,50,87,122,45,81,34,44,34,101,34,58,34,65,81,65$, $66,34,44,34,100,34,58,34,71,82,116,98,73,81,109,104$, $79,90,116,121,115,122,102,103,75,100,103,52,117,95,78$, $45,82,95,109,90,71,85,95,57,107,55,74,81,95,106,110$, $49,68,110,102,84,117,77,100,83,78,112,114,84,101,97$, $83,84,121,87,102,83,78,107,117,97,65,119,110,79,69,98$, $73,81,86,121,49,73,81,98,87,86,86,50,53,78,89,51,121$, $98,99,95,73,104,85,74,116,102,114,105,55,98,65,88,89$, $69,82,101,87,97,67,108,51,104,100,108,80,75,88,121,57$, $85,118,113,80,89,71,82,48,107,73,88,84,81,82,113,110$, $115,45,100,86,74,55,106,97,104,108,73,55,76,121,99$, $107,114,112,84,109,114,77,56,100,87,66,111,52,95,80$, $77,97,101,110,78,110,80,105,81,103,79,48,120,110,117$, $84,111,120,117,116,82,90,74,102,74,118,71,52,79,120$, $52,107,97,51,71,79,82,81,100,57,67,115,67,90,50,118$, $115,85,68,109,115,88,79,102,85,69,78,79,121,77,113,65$, $68,67,54,112,49,77,51,104,51,51,116,115,117,114,89,49$, $53,107,57,113,77,83,112,71,57,79,88,95,73,74,65,88$, $109,120,122,65,104,95,116,87,105,90,79,119,107,50,75$, $52,121,120,72,57,116,83,51,76,113,49,121,88,56,67,49$, 69, 87, 109, 101, 82, 68, 107, 75, 50, 97, 104, 101, 99, 71, 56, 53, $45,111,76,75,81,116,53,86,69,112,87,72,75,109,106,79$, $105,95,103,74,83,100,83,103,113,99,78,57,54,88,53,50$, $101,115,65,81,34,44,34,112,34,58,34,50,114,110,83,79$, $86,52,104,75,83,78,56,115,83,52,67,103,99,81,72,70$, $98,115,48,56,88,98,111,70,68,113,75,117,109,51,115,99$, $52,104,51,71,82,120,114,84,109,81,100,108,49,90,75,57$, $117,119,45,80,73,72,102,81,80,48,70,107,120,88,86,114$, 
$120,45,87,69,45,90,69,98,114,113,105,118,72,95,50,105$, $67,76,85,83,55,119,65,108,54,88,118,65,82,116,49,75$, $107,73,97,85,120,80,80,83,89,66,57,121,107,51,49,115$, $48,81,56,85,75,57,54,69,51,95,79,114,65,68,65,89,116$, $65,74,115,45,77,51,74,120,67,76,102,78,103,113,104,53$, $54,72,68,110,69,84,84,81,104,72,51,114,67,84,53,84$, $51,121,74,119,115,34,44,34,113,34,58,34,49,117,95,82$, $105,70,68,80,55,76,66,89,104,51,78,52,71,88,76,84,57$, $79,112,83,75,89,80,48,117,81,90,121,105,97,90,119,66$, $116,79,67,66,78,74,103,81,120,97,106,49,48,82,87,106$, $115,90,117,48,99,54,73,101,100,105,115,52,83,55,66,95$, $99,111,83,75,66,48,75,106,57,80,97,80,97,66,122,103$, $45,73,121,83,82,118,118,99,81,117,80,97,109,81,117,54$, $54,114,105,77,104,106,86,116,71,54,84,108,86,56,67,76$, $67,89,75,114,89,108,53,50,122,105,113,75,48,69,95,121$, $109,50,81,110,107,119,115,85,88,55,101,89,84,66,55,76$, $98,65,72,82,75,57,71,113,111,99,68,69,53,66,48,102$, $56,48,56,73,52,115,34,44,34,100,112,34,58,34,75,107$, $77,84,87,113,66,85,101,102,86,119,90,50,95,68,98,106$, $49,112,80,81,113,121,72,83,72,106,106,57,48,76,53,120$, $95,77,79,122,113,89,65,74,77,99,76,77,90,116,98,85$, $116,119,75,113,118,86,68,113,51,116,98,69,111,51,90$, 73, 99, 111, 104, 98, 68, 116, 116, 54, 83, 98, 102, 109, 87, 122, $103,103,97,98,112,81,120,78,120,117,66,112,111,79,79$, $102,95,97,95,72,103,77,88,75,95,108,104,113,105,103$, $73,52,121,95,107,113,83,49,119,89,53,50,73,119,106,85$, $110,53,114,103,82,114,74,45,121,89,111,49,104,52,49$, $75,82,45,118,122,50,112,89,104,69,65,101,89,114,104$, $116,116,87,116,120,86,113,76,67,82,86,105,68,54,99,34$, $44,34,100,113,34,58,34,65,118,102,83,48,45,103,82,120$, $118,110,48,98,119,74,111,77,83,110,70,120,89,99,75,49$, $87,110,117,69,106,81,70,108,117,77,71,102,119,71,105$, $116,81,66,87,116,102,90,49,69,114,55,116,49,120,68$, $107,98,78,57,71,81,84,66,57,121,113,112,68,111,89,97$, $78,48,54,72,55,67,70,116,114,107,120,104,74,73,66,81$, $97,106,54,110,107,70,53,75,75,83,51,84,81,116,81,53$, $113,67,122,107,79,107,109,120,73,101,51,75,82,98,66$, $121,109,88,120,107,98,53,113,119,85,112,88,53,69,76$, $68,53,120,70,99,54,70,101,105,97,102,87,89,89,54,51$, $84,109,109,69,65,117,95,108,82,70,67,79,74,51,120,68$, $101,97,45,111,116,115,34,44,34,113,105,34,58,34,108$, $83,81,105,45,119,57,67,112,121,85,82,101,77,69,114,80$, $49,82,115,66,76,107,55,119,78,116,79,118,115,53,69,81$, $112,80,113,109,117,77,118,113,87,53,55,78,66,85,99$, $122,83,99,69,111,80,119,109,85,113,113,97,98,117,57$, $86,48,45,80,121,52,100,81,53,55,95,98,97,112,111,75$, $82,117,49,82,57,48,98,118,117,70,110,85,54,51,83,72$, $87,69,70,103,108,90,81,118,74,68,77,101,65,118,109$, 
$106,52,115,109,45,70,112,48,111,89,117,95,110,101,111$, $116,103,81,48,104,122,98,73,53,103,114,121,55,97,106$, $100,89,121,57,45,50,108,78,120,95,55,54,97,66,90,111$, $79,85,117,57,72,67,74,45,85,115,102,83,79,73,56,34$, $125]$

C.2. JOSE Header

The following example JWE Protected Header declares that:

- the Content Encryption Key is encrypted to the recipient using the PSE2-HS256+A128KW algorithm to produce the JWE Encrypted Key,

- the Salt Input ("p2s") value is $[217,96,147,112,150,117,70$, $247,127,8,155,137,174,42,80,215]$,

o the Iteration count ("p2c") value is 4096,

- authenticated encryption is performed on the plaintext using the AES_128_CBC_HMAC_SHA_256 algorithm to produce the ciphertext and the Authentication Tag, and

o the content type is application/jwk+json.

\{

"alg" : "PBES2-HS256+A128KW", "p2s": "2WCTcJZ1Rvd_CJuJripQ1w", "p2c": 4096 , "enc": "A128CBC-HS256", "cty": "jwk+json" \}

Encoding this JWE Protected Header as BASE64URL (UTF8 (JWE Protected Header)) gives this value (with line breaks for display purposes only):

eyJhbGciOi JQQkVTMi1IUzI INitBMT I S S1 ciLCJwMnMiOi IyVONUY0paMVJ2ZF9DSn VKcmlwUTF 3IiwicDJjI jo0MDk2LCJlbmMiOiJBMTI 4Q0JDLUhTMjU2IiwiY3R5I joi andrK2pzb24ifQ

C.3. Content Encryption Key (CEK)

Generate a 256-bit random Content Encryption Key (CEK). In this example, the value (using JSON array notation) is:

$[111,27,25,52,66,29,20,78,92,176,56,240,65,208,82,112$, $161,131,36,55,202,236,185,172,129,23,153,194,195,48$, $253,182]$ 


\section{C.4. Key Derivation}

Derive a key from a shared passphrase using the PBKDF2 algorithm with HMAC SHA-256 and the specified Salt and Iteration Count values and a 128-bit requested output key size to produce the PBKDF2 Derived Key. This example uses the following passphrase:

Thus from my lips, by yours, my sin is purged.

The octets representing the passphrase are:

$[84,104,117,115,32,102,114,111,109,32,109,121,32,108$, 105, 112, 115, 44, 32, 98, 121, 32, 121, 111, 117, 114, 115, 44, 32, $109,121,32,115,105,110,32,105,115,32,112,117,114,103$, $101,100,46]$

The Salt value (UTF8(Alg) || 0x00 || Salt Input) is:

$[80,66,69,83,50,45,72,83,50,53,54,43,65,49,50,56,75$, $87,0,217,96,147,112,150,117,70,247,127,8,155,137,174$, $42,80,215]$.

The resulting PBKDF2 Derived Key value is: $[110,171,169,92,129,92,109,117,233,242,116,233,170,14$, $24,75]$

\section{C.5. Key Encryption}

Encrypt the CEK with the "A128KW" algorithm using the PBKDF2 Derived Key. The resulting JWE Encrypted Key value is:

$[78,186,151,59,11,141,81,240,213,245,83,211,53,188,134$, $188,66,125,36,200,222,124,5,103,249,52,117,184,140,81$, $246,158,161,177,20,33,245,57,59,4]$

Encoding this JWE Encrypted Key as BASE64URL(JWE Encrypted Key) gives this value:

TrqXOwuNUfDV9VPTNbyGvEJ9JMjefAVn-TR1uIxR9p6hsRQh9Tk7BA

C.6. Initialization Vector

Generate a random 128-bit JWE Initialization Vector. In this example, the value is:

$[97,239,99,214,171,54,216,57,145,72,7,93,34,31,149$, $156]$ 
Encoding this JWE Initialization Vector as BASE64URL(JWE Initialization Vector) gives this value:

Ye9j1qs22DmRSAddIh-VnA

C.7. Additional Authenticated Data

Let the Additional Authenticated Data encryption parameter be ASCII (BASE64URL (UTF8 (JWE Protected Header))). This value is:

$[123,34,97,108,103,34,58,34,80,66,69,83,50,45,72,83$, $50,53,54,43,65,49,50,56,75,87,34,44,34,112,50,115,34$, $58,34,50,87,67,84,99,74,90,49,82,118,100,95,67,74$, $117,74,114,105,112,81,49,119,34,44,34,112,50,99,34,58$, $52,48,57,54,44,34,101,110,99,34,58,34,65,49,50,56,67$, $66,67,45,72,83,50,53,54,34,44,34,99,116,121,34,58,34$, $106,119,107,43,106,115,111,110,34,125]$

\section{C.8. Content Encryption}

Perform authenticated encryption on the plaintext with the AES_128_CBC_HMAC_SHA_256 algorithm using the CEK as the encryption key, the JWE Initialization Vector, and the Additional Authenticated Data value above. The resulting ciphertext is:

$[3,8,65,242,92,107,148,168,197,159,77,139,25,97,42$, $131,110,199,225,56,61,127,38,64,108,91,247,167,150,98$, $112,122,99,235,132,50,28,46,56,170,169,89,220,145,38$, $157,148,224,66,140,8,169,146,117,222,54,242,28,31,11$, $129,227,226,169,66,117,133,254,140,216,115,203,131,60$, $60,47,233,132,121,13,35,188,53,19,172,77,59,54,211$, $158,172,25,60,111,0,80,201,158,160,210,68,55,12,67$, $136,130,87,216,197,95,62,20,155,205,5,140,27,168,221$, $65,114,78,157,254,46,206,182,52,135,87,239,3,34,186$, $126,220,151,17,33,237,57,96,172,183,58,45,248,103,241$, $142,136,7,53,16,173,181,7,93,92,252,1,53,212,242,8$, $255,11,239,181,24,148,136,111,24,161,244,23,106,69,157$, $215,243,189,240,166,169,249,72,38,201,99,223,173,229,9$, $222,82,79,157,176,248,85,239,121,163,1,31,48,98,206$, $61,249,104,216,201,227,105,48,194,193,10,36,160,159$, $241,166,84,54,188,211,243,242,40,46,45,193,193,160,169$, $101,201,1,73,47,105,142,88,28,42,132,26,61,58,63,142$, $243,77,26,179,153,166,46,203,208,49,55,229,34,178,4$, 109, 180, 204, 204, 115, 1, 103, 193, 5, 91, 215, 214, 195, 1, 110, $208,53,144,36,105,12,54,25,129,101,15,183,150,250,147$, $115,227,58,250,5,128,232,63,15,14,19,141,124,253,142$, $137,189,135,26,44,240,27,88,132,105,127,6,71,37,41$, $124,187,165,140,34,200,123,80,228,24,231,176,132,171$, 


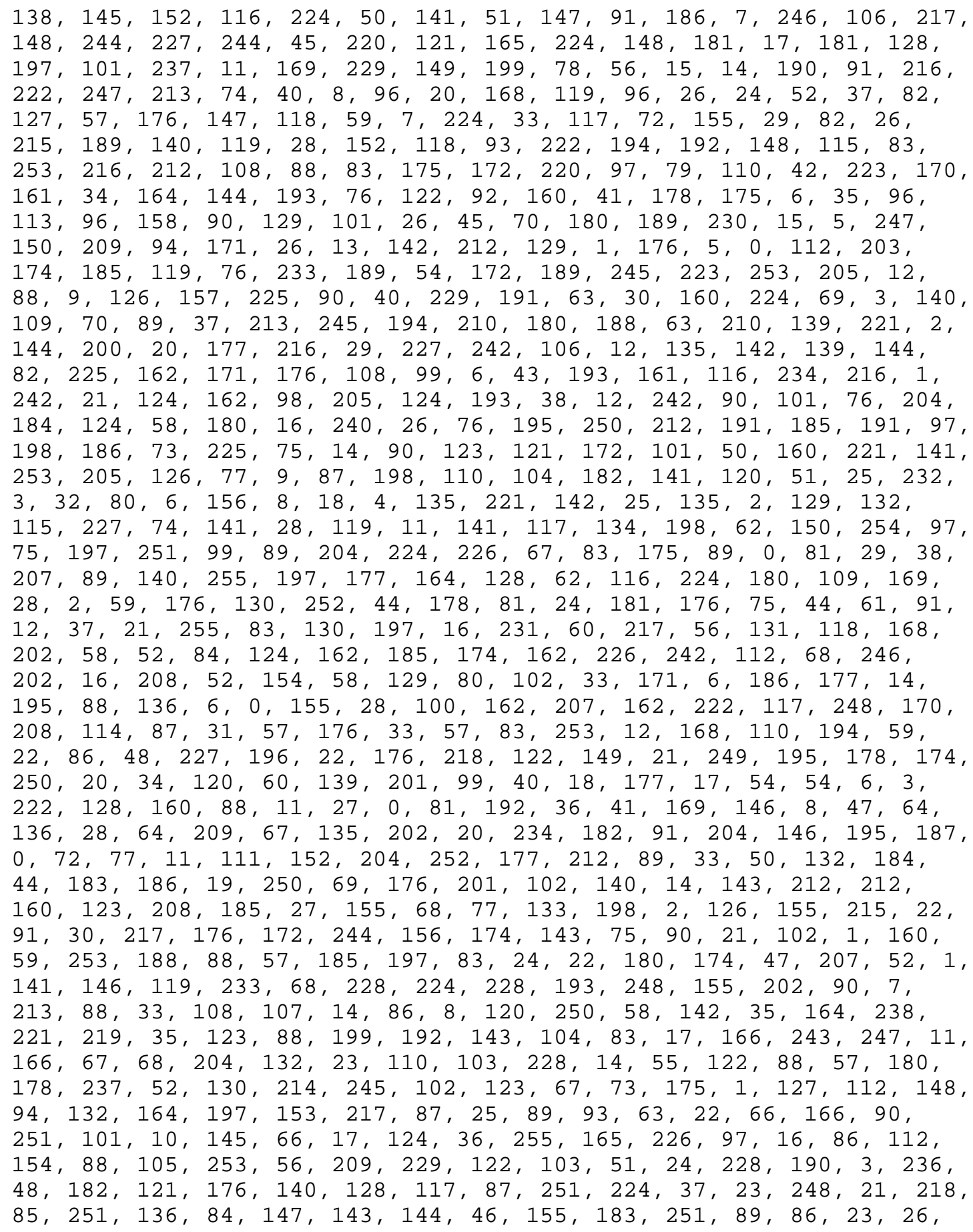


$237,100,167,32,130,173,237,89,55,110,70,142,127,65,230$, $208,109,69,19,253,84,130,130,193,92,58,108,150,42,136$, $249,234,86,241,182,19,117,246,26,181,92,101,155,44,103$, $235,173,30,140,90,29,183,190,77,53,206,127,5,87,8,187$, $184,92,4,157,22,18,105,251,39,88,182,181,103,148,233$, $6,63,70,188,7,101,216,127,77,31,12,233,7,147,106,30$, $150,77,145,13,205,48,56,245,220,89,252,127,51,180,36$, $31,55,18,214,230,254,217,197,65,247,27,215,117,247,108$, $157,121,11,63,150,195,83,6,134,242,41,24,105,204,5,63$, 192, 14, 159, 113, 72, 140, 128, 51, 215, 80, 215, 39, 149, 94, 79, $128,34,5,129,82,83,121,187,37,146,27,32,177,167,71,9$, $195,30,199,196,205,252,207,69,8,120,27,190,51,43,75$, $249,234,167,116,206,203,199,43,108,87,48,155,140,228$, $210,85,25,161,96,67,8,205,64,39,75,88,44,238,227,16$, $0,100,93,129,18,4,149,50,68,72,99,35,111,254,27,102$, $175,108,233,87,181,44,169,18,139,79,208,14,202,192,5$, $162,222,231,149,24,211,49,120,101,39,206,87,147,204$, $200,251,104,115,5,127,117,195,79,151,18,224,52,0,245$, $4,85,255,103,217,0,116,198,80,91,167,192,154,199,197$, $149,237,51,2,131,30,226,95,105,48,68,135,208,144,120$, $176,145,157,8,171,80,94,61,92,92,220,157,13,138,51,23$, $185,124,31,77,1,87,241,43,239,55,122,86,210,48,208$, $204,112,144,80,147,106,219,47,253,31,134,176,16,135$, 219, 95, 17, 129, 83, 236, 125, 136, 112, 86, 228, 252, 71, 129, 218, $174,156,236,12,27,159,11,138,252,253,207,31,115,214$, $118,239,203,16,211,205,99,22,51,163,107,162,246,199,67$, $127,34,108,197,53,117,58,199,3,190,74,70,190,65,235$, $175,97,157,215,252,189,245,100,229,248,46,90,126,237,4$, $159,128,58,7,156,236,69,191,85,240,179,224,249,152,49$, $195,223,60,78,186,157,155,217,58,105,116,164,217,111$, $215,150,218,252,84,86,248,140,240,226,61,106,208,95,60$, $163,6,0,235,253,162,96,62,234,251,249,35,21,7,211,233$, $86,50,33,203,67,248,60,190,123,48,167,226,90,191,71$, $56,183,165,17,85,76,238,140,211,168,53,223,194,4,97$, $149,156,120,137,76,33,229,243,194,208,198,202,139,28$, $114,46,224,92,254,83,100,134,158,92,70,78,61,62,138$, $24,173,216,66,198,70,254,47,59,193,53,6,139,19,153$, 253, 28, 199, 122, 160, 27, 67, 234, 209, 227, 139, 4, 50, 7, 178, $183,89,252,32,128,137,55,52,29,89,12,111,42,181,51$, $170,132,132,207,170,228,254,178,213,0,136,175,8]$

The resulting Authentication Tag value is:

$[208,113,102,132,236,236,67,223,39,53,98,99,32,121,17$, $236]$ 
Encoding this JWE Ciphertext as BASE64URL (JWE Ciphertext) gives this value (with line breaks for display purposes only):

AwhB8 lxrlKjFn 02 LGWEqg 27 H 4 Tg 9 fyZAbFv3p5Zi cHpj64 QyHC 44 qql Z3 JEmnZTgQo wIqZJ13 jbyHB8 LgeP i qUJ1 h f 6M2 HP Lg zw8 L-mEeQ0 jvDUTrE0 7NtOerBk8bwBQyZ6g 0kQ3DEOIglfYxV8-FJvNBYwbqN1Bck6d_i70t jSHV-8DIrp-3JcRIe05YKy30i34Z_ GOiAc1EK21B11C_AE11PII_wVvtRiUiG8YofQXakWd1_098Kap-UgmyWPfreUJ31JP nbD 4Ve95owEfMGLOPflo2Mn jaTDCwQokoJ_xplQ2vNP z 8 iguLcHBoKl lyQFJL2mOWB wqhBo $90 j-0800$ as 5mmLsvQMT f I Ir IEbbTMz HMBZ 8EFW9 fWwwFu ODWQJGkMNhmBZQ-3 lvqTC-M6-gWA6D8PDhONfP2Oib2 HGi zwG1 iEaX8GRyUpfLul jCLIe1DkGOewhKuKkZ h0 4DKNM5Nbugf2atmU9OP 0 Ldx5peCUtRG1 gMV1 7Qup5 ZXHT jgPDr5b2N731UooCGAU qHdgGhg0JVJ_ObCTdjsH4CF1SJsdUhrXvYx3HJh2Xd7CwJRzU_3Y1GxYU6-s3GFPbi rfqqEipJDBTHpcoCmyrwYjYHFgnlqBZRotRrS95g 8F 95bRXqsaDY7UgQGwBQBwy 665 d0zpvTasvfXf_c0MWA1-neFaKOW_Px6g4EUDjG1GWSXV9cLStLW_0ovdApDIFLHYHe PyagyH jouQUuGiq7BsYwYrwaF 06tgB8hV8omLNfMEmDP JaZUzMuHw6tBDwGkzD-tS_ ub 9hxrpJ4UsOWnt 5 rGUyoN2N_c1-TQ1Xxm5oto14MxnoAyBQBpwIEgSH3Y4ZhwKBhH P jSo 0 cdwuNdYbGPpb-YUvF-2NZ zODiQ1OvWQBRHSbPWYz_xbGkgD 504 LRt qRwCO 7 CC _CYYURi1sEssPVsMJRX_U4LFEOc82TiDdqjKOjRUfKK5rqLi8nBE9soQODSaOoFQZi GrBrqxDsNYiAYAmxxkos-i3nX4qtByVx85sCE5U_OMqG7COxZWMOPEFrDaepUV-cOY rvoUIng 8 i 81 jKBKxETY2BgPegKBYCxsAUCAkKamSCC9AiBxA0UOHyhTqt l vMksO7AE hNC2-YzPyx1FkhMoS4LLe6E_pFsMlmjA6P1NSge9C5G5tETYXGAn6b1xZbHtmwrPSC ro9LWhVmAaA7_bxYObnFUxgWtK4vzzQBjZJ36UTk40TB-JvKWg fVWCFsaw5WCH j600 4 jpo 7d2yN7WMfAj2hTEabz 9wumQ0 TMhBduZ-QON3pYObSy7TSC1vVme0NJrwF_cJRe hKTFmdlXGVldPxZCplr7ZQqRQhF $8 \mathrm{JP}-14 \mathrm{mEQVnCaWGn9ONH} \mathrm{lemczGOS-A-wwt} \mathrm{nmw} \mathrm{j}$ B1V_vgJRf4FdpV-4hUk 4-QLpu3-1lWFxrtZKcggq3tWTduRo5_eebebUUT_VSCgsFC OmyWKoj56lbxthN1 9hq1XGWbLGfrrR6MWh23vk01 zn8FVwi 7uFwEnRYSafsnWLa1Z5 TpBj9GvAdl2H9NHw zpB5NqHpZNkQ3NMDj13Fn8fzO0JB83Etbm_tnFQfcb13X3bJ15 Cz-Ww1MGhvIpGGnMBT_ADp9xSIyAM9dQ1yeVXk-AIgWBUlN5uyWSGyCxp0 cJwx 7 HxM 38 z $0 U I$ IeBu-Myt L-eqndM7 Lxyt sVzCb jOTSVRmhYEMI zUAnS1gs 7uMQAGRdgRIEITJE SGMjb_4bZq9s6Ve1LKkSi 0_QDsrABaLe55UY0 zF 4 ZS fOV5PMyPtocwV_dcNP IxLgNA D1BFX_Z9kAdMZQW6 fAms fF le0 zAoMe 419 pMESH0 JB 4 s JGdCKtQXj1 cXNydDYozF 718 H00BV_Er7zd6VtIw0MxwkFCTatsv_R-GsBCH218RgVPsfYhwVuT 8R4HarpzsDBufC4 r8_c8fc97278sQ081jFjOja6L2x0N_ImzFNXU6xwO-Ska-QeuvYZ3X_L31ZOX4Llp7QSfgDoHnOxFv1Xws-D5mDHD3 zxOup2b2 TppdKTZb9eW2vxUVviM8OI 9at BfP KMGAO v9omA-6vv5 IxUH0-1WMi HLQ_g8vnswp-Jav0c4t6URVUzu jNOoNd_CBGGVnHiJTCHI 88LQxSqLHHIu 4Fz-U2SGnlxGTj0-ihit2ELGRv4vO8E1Bos Tmf0cx3qgG0Pq0eOLBD IHsrdz_CCAi Tc0HVkMbyq1M6qEhM-q5P 6y1QCIrwg

Encoding this JWE Authentication Tag as BASE64URL(JWE Authentication Tag) gives this value:

0HFmhOzSQ98nNWJjIHkR7A 


\section{C.9. Complete Representation}

Assemble the final representation: The JWE Compact Serialization of this result, as defined in section 7.1 of [JWE], is the string BASE64URL (UTF8 (JWE Protected Header)) ||' '' || BASE64URL (JWE Encrypted Key) || '.' || BASE64URL (JWE Initialization Vector) ||' .' || BASE64URL (JWE Ciphertext) ||' .' || BASE64URL (JWE Authentication Tag). 
The final result in this example (with line breaks for display purposes only) is:

eyJhbGciOi JQQkVTMi 1 IUzI INitBMT I 4S1 ciLCJwMnMiOi IyV0NUY0paMVJ2ZF 9DSn VKcmlwUTF 3 I iwicDJjI jo0MDk2LCJlbmMiOi JBMT I 4 Q JDLUhTMjU2IiwiY3R5Ijoi andrK2pzb24ifQ. TrqXOwuNUfDV9VPTNbyGvEJ9JMjefAVn-TR1uIxR9p6hsRQh9Tk7BA. Ye9j1qs22DmRSAddIh-VnA. AwhB8 lxrlKjFn 02 LGWEqg 27 H 4 Tg 9 fyZAbFv3p5Zi cHpj 64 QyHC 44 qql Z3 JEmnZTgQo wIqZJ13 jbyHB8 LgeP i qUJ1h f 6M2 HP Lg zw 8 L-mEeQ0 jvDUTrE0 7NtOerBk8bwBQyZ6g 0kQ3DEOIglfYxV8-FJvNBYwbqN1Bck6d_i70t jSHV-8DIrp-3JcRIe05YKy30i34Z_ GOiAc1EK21B11C_AE11PII_wVvtRiUiG8YofQXakWd1_098Kap-UgmyWPfreUJ31JP nbD 4Ve95 owE fMGLOP flo2Mn jaTDCwQokoJ_xplQ2vNP z 8 iguLcHBoKl lyQF JL2mOWB wqhBo $90 j-0800$ as 5mmL svQMT f I IrIEbbTMz HMBZ 8EFW9 fWwWF u ODWQJGkMNhmBZQ-3 lvqTC-M6-gWA6D 8PDhONfP2Oib2HGi zwG1 i EaX8GRyUpfLul jCLIe1DkGOewhKuKkZ h0 4DKNM5Nbugf2atmU9OP 0 Ldx5peCUtRG1 gMVI 7Qup5ZXHT jgPDr5b2N731UooCGAU qHdgGhg0JVJ_ObCTdjsH4CF1SJsdUhrXvYx3HJh2Xd7CwJRzU_3Y1GXYU6-s3GFPbi rfqqEipJDBTHpcoCmyrwYjYHFgnlqBZRotRrS95g 8F 95bRXqsaDY7UgQGwBQBwy 665 d0zpvTasvfXf_c0MWAl-neFaKOW_Px6g4EUDjG1GWSXV9cLStLw_0ovdApDIFLHYHe PyagyH jouQUuGiq7BsYwYrwaF 0 6tgB8hV8omLNfMEmDP JaZUzMuHw6tBDwGkzD-tS_ ub9hxrpJ4UsOWnt 5 rGUyoN2N_c1-TQ1Xxm5 oto14MxnoAyBQBpwIEgSH3Y4ZhwKBhH P jSo 0 cdwuNdYbGPpb-YUvF-2NZzODiQ1OvWQBRHSbPWYz_xbGkgD50 4 LRtqRwCO7CC _CyYURi1sEssPVsMJRX_U4LFEOC82TiDdqjKOjRUfKK5rqLi8nBE9soQODSaOoFQZi GrBrqxDsNYiAYAmxxkos-i3nX4 qtByVx85sCE5U_OMqG7COxZWMOPEFrDaepUV-cOy rvoUIng 8 i 81 jKBKxETY2BgPegKBYCXSAUCAkKamSCC9AiBxA0UOHyhTqt l vMksO7AE hNC2-YzPyx1FkhMoS4LLe6E_pFsMlmjA6P1NSge9C5G5tETYXGAn6b1xZbHtmwrPSC ro9LWhVmAaA7_bxYObnFUxgWtK4vzzQBjZJ36UTk40TB-JvKWgfVWCF saw5WCH j600 4 jpo7d2yN7WMfAj2hTEabz 9wumQ0 TMhBduZ-QON3pYObSy7TSC1vVme0NJrwF_cJRe hKTFmdlXGVldPxZCplr7ZQqRQhF $8 \mathrm{JP}-14 \mathrm{mEQVnCaWGn} 90 \mathrm{NH}$ lemczGOS-A-wwt nmw jI B1V_vgJRf 4FdpV-4hUk4-QLpu3-11WFxrtZKcggq3tWTduRo5_QebQbUUT_VSCgsFC OmyWKoj56lbxthN1 9hq1XGWbLGfrrR6MWh23vk01 zn8FVwi 7uFwEnRYSafsnWLa1z5 TpB j9GvAdl2H9NHw zpB5NqHpZNkQ3NMD j13Fn 8 f zO0 JB83Etbm_tnFQfcb13X3bJ15 Cz-Ww1MGhvIpGGnMBT_ADp9xSIyAM9dQ1yeVXk-AIgWBUlN5uyWSGyCxp0 cJwx 7 HxM 38 z 0UIeBu-MytL-eqndM7 LxytsVzCb jOTSVRmhYEMI zUAnS1gs 7uMQAGRdgRIElT JE SGMjb_4bZq9s6Ve1LKkSi 0_QDsrABaLe55UY0 zF 4 ZS fOV5PMyP tocwV_dcNP l xLgNA D1BFX_z9kAdMZQW6 fAms fF le0 zAoMe 419 pMESH0 JB 4 s JGdCKtQXj1 cXNydDYozF 718 H00BV_Er7zd6VtIw0MxwkFCTatsv_R-GsBCH218RgVPsfYhwVuT8R4 HarpzsDBufC4 r8_c8fc97278sQ081jFjOja6L2x0N_ImzFNXU6xwO-Ska-QeuvYZ3X_L31ZOX4Llp7QSfgDoHnOxFv1Xws-D 5mDHD 3 zxOup2b2 TppdKTZb9ew2vxUVviM8OI 9 at BfP KMGAO v9omA-6vv5 IxUH0-1WMi HLQ_g8vnswp-Jav0c4t6URVUzujNOoNd_CBGGVnHiJTCH 1 88LQxSqLHHIu 4Fz-U2SGnlxGT j0-ihit2ELGRv4vO8E1Bos Tmf0 cx3qgG0Pq0eOLBD IHsrdz_CCAiTc0HVkMbyq1M6qEhM-q5P 6y1QCIrwg.

0HFmhOzSQ98nNWJjIHkR7A 


\section{Acknowledgements}

A JSON representation for RSA public keys was previously introduced by John Panzer, Ben Laurie, and Dirk Balfanz in Magic Signatures [MagicSignatures].

Thanks to Matt Miller for creating the encrypted key example and to Edmund Jay and Brian Campbell for validating the example.

This specification is the work of the JOSE working group, which includes dozens of active and dedicated participants. In particular, the following individuals contributed ideas, feedback, and wording that influenced this specification:

Dirk Balfanz, Richard Barnes, John Bradley, Brian Campbell, Breno de Medeiros, Stephen Farrell, Joe Hildebrand, Edmund Jay, Stephen Kent, Ben Laurie, James Manger, Matt Miller, Kathleen Moriarty, Chuck Mortimore, Tony Nadalin, Axel Nennker, John Panzer, Eric Rescorla, Pete Resnick, Nat Sakimura, Jim Schaad, Ryan Sleevi, Paul Tarjan, Hannes Tschofenig, and Sean Turner.

Jim Schaad and Karen O'Donoghue chaired the JOSE working group and Sean Turner, stephen Farrell, and Kathleen Moriarty served as Security Area Directors during the creation of this specification.

Author's Address

Michael B. Jones

Microsoft

EMail: mbj@microsoft.com

URI: http://self-issued.info/ 